January 2006

CERN-PH-TH/2005-260

\title{
The Well-Tempered Neutralino
}

\author{
N. Arkani-Hamed ${ }^{1}$, A. Delgado ${ }^{2}$, G.F. Giudice ${ }^{2}$ \\ ${ }^{1}$ Jefferson Laboratory of Physics, Harvard University, \\ Cambridge, Massachusetts 02138, USA \\ ${ }^{2}$ CERN, Theory Division, CH-1211 Geneva 23, Switzerland
}

\begin{abstract}
The dark-matter prediction is usually considered as one of the successes of lowenergy supersymmetry. We argue that, after LEP constraints are taken into account, the correct prediction for the dark-matter density, at a quantitative level, is no longer a natural consequence of supersymmetry, but it requires special relations among parameters, highly sensitive to small variations. This is analogous to the problem of electroweak-symmetry breaking, where the correct value of the $Z$ mass is obtained only with a certain degree of fine tuning. In the general parameter space of low-energy supersymmetry, one of the most plausible solution to reproduce the correct value of the dark-matter density is the well-tempered neutralino, which corresponds to the boundary between a pure Bino and a pure Higgsino or Wino. We study the properties of well-tempered neutralinos and we propose a simple limit of split supersymmetry that realizes this situation.
\end{abstract}




\section{The Supersymmetric Dark Matter Impasse}

The natural prediction of thermal-relic dark matter is usually considered as one of the most attractive features of models with low-energy supersymmetry. Indeed a stable, neutral, colourless, weakly-interacting particle with Fermi-scale mass leads to a present dark-matter density in rough agreement with observations [1, 2]

$$
\Omega_{\mathrm{DM}} h^{2}=0.113 \pm 0.009
$$

quite independently of any detail of the cosmological evolution at temperatures higher than the freeze-out temperature $T_{f} \sim 1-100 \mathrm{GeV}$. Low-energy supersymmetry with conserved R-parity gives a satisfactory theoretical framework for the existence of such a particle [3].

Here we want to argue that, although dark matter was certainly a natural prediction of supersymmetry in the pre-LEP epoch, it is generically no longer true, at a quantitative level, after LEP data are taken into account. To illustrate the problem, let us consider the supersymmetric extension of the Standard Model with minimal field content, and with general soft terms. An acceptable thermal dark-matter candidate is obtained in the case in which a neutralino is the lightest supersymmetric particle (LSP). As LEP data are forcing the soft terms to be typically larger than $M_{Z}$, a description of the neutralino in terms of current eigenstates is becoming increasingly appropriate. We will then start our discussion with the case in which the neutralino is pure Bino, Wino or Higgsino and later generalize to mixed states.

\subsection{Bino}

The Bino is a gauge singlet whose annihilation in the early universe occurs through squark and slepton exchange. Since sleptons are usually lighter than squarks and right-handed sleptons have the largest hypercharge, the Bino annihilation cross section and its contribution to the present $\Omega$ are well approximated by

$$
\begin{gathered}
\left\langle\sigma_{\tilde{B}} v\right\rangle=\frac{3 g^{4} \tan ^{4} \theta_{W} r\left(1+r^{2}\right)}{2 \pi m_{\tilde{e}_{R}}^{2} x(1+r)^{4}}, \quad x \equiv \frac{M_{1}}{T}, \quad r \equiv \frac{M_{1}^{2}}{m_{\tilde{e}_{R}}^{2}} \\
\Omega_{\tilde{B}} h^{2}=1.3 \times 10^{-2}\left(\frac{m_{\tilde{e}_{R}}}{100 \mathrm{GeV}}\right)^{2} \frac{(1+r)^{4}}{r\left(1+r^{2}\right)}\left(1+0.07 \log \frac{\sqrt{r} 100 \mathrm{GeV}}{m_{\tilde{e}_{R}}}\right) .
\end{gathered}
$$

Here $M_{1}$ is the Bino mass and $m_{\tilde{e}_{R}}$ is the mass of any of the three degenerate right-handed sleptons. The value of $\Omega_{\tilde{B}} h^{2}$ as a function of $M_{1}$ is shown in fig. 1 for $M_{1} / m_{\tilde{e}_{R}}$ varying between 0.9 and 0.3 . For $r$ very close to 1 , co-annihilation becomes important, and eq. (3) 
is no longer valid. We will discuss co-annihilation later, and for the moment we assume $M_{1} / m_{\tilde{e}_{R}}<0.9$. Then, requiring that dark matter is constituted by relic Binos, we find that eq. (11) implies $m_{\tilde{e}_{R}}<111 \mathrm{GeV}$ at $95 \% \mathrm{CL}$. Present LEP limits 4 require $m_{\tilde{e}_{R}}>100 \mathrm{GeV}$, $m_{\tilde{\mu}_{R}}>97 \mathrm{GeV}, m_{\tilde{\tau}_{R}}>93 \mathrm{GeV}$ at $95 \% \mathrm{CL}$ (for neutralino mass of $40 \mathrm{GeV}$ ). Therefore, while before LEP a pure Bino appeared as an adequate dark-matter candidate in broad range of parameters, after LEP the typical prediction is that the Bino relic density is too large.

As it is well known, agreement with dark-matter observation can still be achieved under special conditions on the supersymmetry soft terms. One well-studied possibility [5] is that the Bino and the lightest slepton (usually the stau) are nearly degenerate in mass, so that co-annihilation [6] reduces the relic abundance. This requires a rather precise correlation among parameters, $\left(m_{\tilde{\tau}}-M_{1}\right) / M_{1} \lesssim T_{f} / M_{1} \sim 5 \%$. Another possibility to reduce the relic abundance is to exploit an almost-resonant $s$-channel annihilation [7]. This requires $\mid m_{H}-$ $2 M_{1} \mid / m_{H} \ll 1$, where $m_{H}$ is the mass of the CP-odd or the heavy CP-even Higgs boson. The occurrance of resonant light-Higgs exchange is almost ruled out by data.

There are also alternative ways to reduce the Bino relic density, which however depend somehow on the cosmological history. If the reheat temperature after a period of entropy production $T_{R H}$ is lower than the freeze-out temperature $T_{f}$, than the actual dark-matter relic density can be much smaller than what is estimated by the usual thermal calculation [8]. In this case, the final abundance has a steep dependence on $T_{R H}$.

A different possibility is to assume that the Bino is only the next-to-lightest supersymmetric particle (NLSP), allowing for its decay. If the true LSP is sufficiently weakly coupled to have a negligible thermal relic abundance, but strongly-enough coupled for the Bino decay to be cosmologically harmless, then $\Omega_{\mathrm{LSP}}=\left(m_{\mathrm{LSP}} / M_{1}\right) \Omega_{\tilde{B}}$ can be reconciled with data, for an appropriate value of $m_{\mathrm{LSP}}$. The gravitino is the most motivated candidate for this scenario [9, 10, but the more exotic cases of axino or modulino have also been considered [11]. A difficulty often encountered in this approach is that the late Bino decay can upset the usual nucleosynthesis predictions. Take the case of a gravitino with mass $m_{\tilde{G}}$. If we require that the Bino decay occurs before the onset of nucleosynthesis, then $m_{\tilde{G}}<\left(M_{1} / 100 \mathrm{GeV}\right)^{5 / 2} 10 \mathrm{MeV}$. To obtain a sufficient $\Omega_{\tilde{G}}$ from Bino decay with such a low value of $m_{\tilde{G}}$, it is necessary to have $m_{\tilde{e}_{R}}>2 \mathrm{TeV}\left(M_{1} / 100 \mathrm{GeV}\right)^{1 / 8}$. Otherwise the impact of the decay on nucleosynthesis has to be carefully studied, and indeed the case of a NLSP neutralino is excluded [9]. The situation is better for a $\tilde{\tau}$ NLSP [9], but a recent analysis [10] claims that, in the context of the minimal supergravity model, a dark-matter gravitino coming from $\tilde{\tau}$ decay is also excluded. A way out is to rely on a thermal population of gravitinos, but then the relic-abundance prediction is sensitive on initial cosmological conditions, through $T_{R H}$. 


\subsection{Higgsino}

The Higgsino is traditionally considered a less favourable dark-matter candidate, because it is not the LSP in constrained supersymmetric models and because its annihilation cross section is very efficient. The first argument is very much tied to the assumption of universality where a large Higgsino mass $\mu$ is necessary to cancel the positive contribution to $M_{Z}^{2}$ from all other soft terms, in the radiative electroweak mechanism. Smaller values of $\mu$ occur in the so-called hyperbolic branch 12 and focus point 13 . However, as soon as we depart from universality, the request of large $\mu$ is no longer justified. Consider for instance the case in which the soft mass terms for the stops and for the Higgses at the cut-off scale are different. Since, for sufficiently high cut-off, the stops give a positive contribution to $M_{Z}^{2}$ and the Higgses give a negative contribution, an appropriate cancellation can be obtained for any value of $\mu$.

In the limit of pure Higgsino, the dominant annihilation channel is into gauge bosons. Since charged and neutral Higgsino states are nearly mass-degenerate, co-annihilation is essential [14. When $\mu$ is larger than $M_{W}$, the effective annihilation cross section and the Higgsino contribution to $\Omega$ are well approximated by (see appendix A)

$$
\begin{gathered}
\left\langle\sigma_{e f f} v\right\rangle=\frac{g^{4}}{512 \pi \mu^{2}}\left(21+3 \tan ^{2} \theta_{W}+11 \tan ^{4} \theta_{W}\right) \\
\Omega_{\tilde{H}} h^{2}=0.10\left(\frac{\mu}{1 \mathrm{TeV}}\right)^{2}
\end{gathered}
$$

where in $\Omega_{\tilde{H}}$ we have suppressed a small logarithmic dependence on $\mu$. The annihilation into $\bar{t} t$ pairs through stop exchange is less important, as it is evident from fig. 1, where we show the Higgsino relic abundance: the narrow band corresponds to a variation of the stop mass from $m_{\tilde{t}}=1.5 \mu$ to infinity. In the mass region allowed by LEP ( $\mu \gtrsim 100 \mathrm{GeV}$ ), we have the opposite problem of the Bino: the relic abundance is too low, unless $\mu$ is about 1 $\mathrm{TeV}$. Such large values of $\mu$ require a significant fine-tuning in order to reproduce the weak scale and are at odds with the original motivation of supersymmetry. They are acceptable in the case of Split Supersymmetry [15, 16, 17, where supersymmetry is not required to solve the hierarchy problem. Moreover, such large values of the LSP mass typically bring the supersymmetric spectrum beyond the reach of the LHC.

As in the case of the Bino, by invoking solutions that depend on initial cosmological conditions, we can obtain a correct Higgsino abundance with lower values of $\mu$, . Since now we want to increase the Higgsino abundance, rather than depleting it, we have to assume that the super-weakly interacting relic (gravitinos or other exotic particles) is not the LSP, but it decays into Higgsinos. The decay process has to occur after Higgsinos have decoupled, 


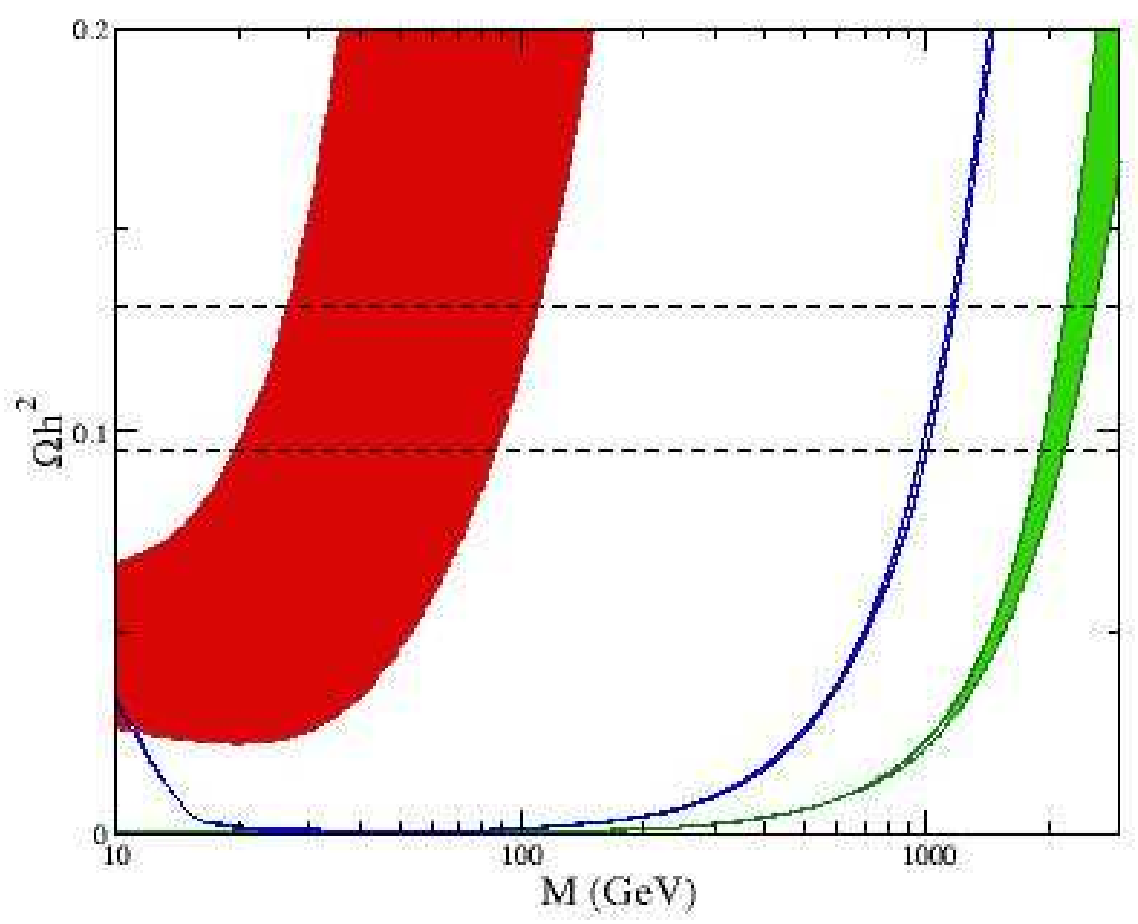

Figure 1: The three bands show the contribution to $\Omega h^{2}$ from pure Bino LSP with $0.3<$ $M_{1} / m_{\tilde{e}_{R}}<0.9$ (red band), Higgsino LSP with $1.5<m_{\tilde{t}} / \mu<\infty$ (blue band) and Wino LSP with $1.5<m_{\tilde{\ell}_{L}} / M_{2}<\infty$ (green band).

but early enough not to upset the nucleosynthesis predictions. The final relic abundance will of course depend on the initial gravitino density or, ultimately, on $T_{R H}$.

\subsection{Wino}

The Wino can be the LSP in anomaly mediation [18, 19. In the case of pure state, the dominant annihilation is into gauge bosons, with a contribution from fermion-antifermion channel through scalar exchange. Coannihilation among the different states in the Wino weak triplet is important. In the limit in which the Wino mass $M_{2}$ is larger than $M_{W}$, the effective annihilation cross section and the Wino contribution to $\Omega$ are well approximated by (see appendix A)

$$
\begin{gathered}
\left\langle\sigma_{e f f} v\right\rangle=\frac{3 g^{4}}{16 \pi M_{2}^{2}}, \\
\Omega_{\tilde{W}} h^{2}=0.13\left(\frac{M_{2}}{2.5 \mathrm{TeV}}\right)^{2} .
\end{gathered}
$$


In $\Omega_{\tilde{W}}$ we have suppressed a small logarithmic dependence on $M_{2}$. The Wino relic abundance is shown in fig. 1, where the narrow band corresponds to varying the left slepton masses from $m_{\tilde{\ell}_{L}}=1.5 M_{2}$ to infinity, and taking the other supersymmetric scalars very heavy. The situation is analogous to the Higgsino case, and actually it is even more extreme, because values of $M_{2}$ as large as $2.5 \mathrm{TeV}$ are required to reproduced the observed value of the darkmatter density. In the case of anomaly mediation, the value of the gravitino mass is adequate to generate an acceptable non-thermal $\tilde{W}$ population to account for the dark matter, from gravitino or moduli decay [20, 21].

\subsection{The Dark-Matter Impasse and the Well-Tempered Neutralino}

Figure 1 illustrates the meaning of the supersymmetric dark-matter impasse. Before LEP, values of $M_{1,2}$ and $\mu$ below $M_{Z}$ were allowed and supersymmetry could explain the weak

scale without much fine tuning. At that time, a description of neutralinos in terms of pure states was not appropriate, and the LSP was naturally a mixture. Its relic abundance cannot be directly read from fig. [1 because more annihilation channels are possible for mixed states. However, it is clear from fig. 田 that the correct value of $\Omega_{\mathrm{DM}}$ could be reproduced, for mixed states and with light sleptons, in a broad range of parameters. Certainly the prediction for $\Omega_{\mathrm{DM}}$ could vary significantly, but the crucial point is that this variation did not have a critical behaviour with the underlying soft terms. On the other hand, the options left open after LEP, such as $\tilde{\tau}$-coannihilation or Higgs-resonance, give a prediction of $\Omega_{\mathrm{DM}}$ which is critically sensitive on some soft parameters.

The supersymmetric dark-matter impasse is very similar to the naturalness problem. Before LEP, the prediction for $M_{Z}$ could certainly vary with the parameters, and the physical value could be obtained only for particular choices. However, the physical value of $M_{Z}$ did not imply a special critical sensitivity on soft parameters. After LEP, the correct value of $M_{Z}$ can still be reproduced, but the result has, at best, few-percent sensitivity on parameter variations. As the soft terms are varied within the experimentally-allowed region, in almost all cases one finds $M_{Z}=0$ (no electroweak breaking) or $M_{Z} \gtrsim \mathrm{TeV}$. The physical value of $M_{Z}$ is obtained only for critical values of the soft terms, and this requires a certain amount of fine tuning.

As we vary the values of the soft terms in the experimentally-allowed region without accepting excessive fine tunings, we are finding that $\Omega_{\mathrm{DM}}$ is typically too large (Bino LSP) or too small (Higgsino or Wino LSP). Since $\Omega_{\mathrm{DM}}$ is a continuous function of parameters, it is clear that, along the critical lines that separates $\tilde{B} / \tilde{H}$ LSP and $\tilde{B} / \tilde{W}$ LSP, we can 
obtain the correct value of the dark-matter density. This may appear as a fine-tuning but, if supersymmetry is responsible for dark matter, LEP has forced us towards such tuned regions. If we consider the general supersymmetric parameter space, without bias in favour of universal/unified soft terms, these regions appear as some of the most plausible option for dark matter, still allowed after LEP.

We will therefore consider in this paper the case in which the LSP neutralino is mass degenerate or maximally mixed with other states (either $\tilde{B} / \tilde{H}$ or $\tilde{B} / \tilde{W}$ ), although the relevant soft terms are typically larger than $M_{Z}$. This requires a precise relation among parameters $\left(\left|M_{1}\right| \simeq|\mu|\right.$ or $\left.\left|M_{1}\right| \simeq\left|M_{2}\right|\right)$ and therefore we will refer to this case as the "well-tempered neutralino". We will start with a general classification, but many considerations about mixed or degenerate heavy neutralinos have already been made in the literature. The case of the $\tilde{B} / \tilde{H}$ tempered neutralino was studied in the context of focus-point [22], non-universal soft terms [23], and Split Supersymmetry [16, 24, 25]. The case of $\tilde{B} / \tilde{W}$ tempered neutralino was previously considered in refs. [25, 26, 27], assuming non-minimal gaugino mass relations. We will present here a class of theories where the relation $\left|M_{1}\right| \simeq\left|M_{2}\right|$ can be obtained with conventional gaugino-mass boundary conditions, like anomaly mediation or gaugino unification.

\section{The Well-Tempered Bino/Higgsino}

In the case of the well-tempered $\tilde{B} / \tilde{H}$, the Wino can be decoupled and the effective $3 \times 3$ neutralino mass matrix in the basis $\tilde{B}, \tilde{H}_{1} \equiv\left(\tilde{H}_{u}-\tilde{H}_{d}\right) / \sqrt{2}, \tilde{H}_{2} \equiv\left(\tilde{H}_{u}+\tilde{H}_{d}\right) / \sqrt{2}$ is

$$
\begin{gathered}
\mathcal{M}=\left(\begin{array}{ccc}
M_{1} & -\frac{s_{\beta}+c_{\beta}}{\sqrt{2}} s_{W} M_{Z} & \frac{s_{\beta}-c_{\beta}}{\sqrt{2}} s_{W} M_{Z} \\
-\frac{s_{\beta}+c_{\beta}}{\sqrt{2}} s_{W} M_{Z} & \mu & 0 \\
\frac{s_{\beta}-c_{\beta}}{\sqrt{2}} s_{W} M_{Z} & 0 & -\mu
\end{array}\right) \\
-\frac{M_{W}^{2}}{2 M_{2}}\left(\begin{array}{ccc}
0 & 0 & 0 \\
0 & 1+s_{2 \beta} & c_{2 \beta} \\
0 & c_{2 \beta} & 1-s_{2 \beta}
\end{array}\right)+\mathcal{O}\left(\frac{1}{M_{2}^{2}}\right) .
\end{gathered}
$$

Here and in the following $s_{\beta} \equiv \sin \beta, c_{\beta} \equiv \cos \beta, s_{W} \equiv \sin \theta_{W}$, etc. The general discussion of the diagonalization of $\mathcal{M}$, including the effect of the CP-violating phase, is given in appendix B. For real $M_{1}$ and $\mu$, the well-tempered condition can be achieved in two cases: $M_{1} \simeq \mu$ and $M_{1} \simeq-\mu$. When $\left|M_{1}\right|,|\mu|$ and their difference are larger than $M_{Z}$, the eigenvalues of $\mathcal{M}$, dropping terms suppressed by $M_{1}$, are approximately given by

$$
M_{1}+\theta_{ \pm}^{2}\left(M_{1} \mp \mu\right), \quad \pm \mu-\theta_{ \pm}^{2}\left(M_{1} \mp \mu\right), \quad \mp \mu,
$$




$$
\theta_{ \pm}=\frac{\left(s_{\beta} \pm c_{\beta}\right) s_{W} M_{Z}}{\sqrt{2}\left(\mu \mp M_{1}\right)},
$$

where the \pm sign refers to the cases $\mu \simeq \pm M_{1}$. The orthogonal matrix $N$ that makes $N \mathcal{M} N^{T}$ diagonal is

$$
N=\left(\begin{array}{ccc}
1-\frac{\theta_{+}^{2}}{2}-\frac{\theta_{-}^{2}}{2} & \theta_{+} & \theta_{-} \\
-\theta_{+} & 1-\frac{\theta_{+}^{2}}{2} & -\theta_{+} \theta_{-} \frac{\left(M_{1}+\mu\right)}{2 \mu} \\
-\theta_{-} & \theta_{+} \theta_{-} \frac{\left(M_{1}-\mu\right)}{2 \mu} & 1-\frac{\theta_{-}^{2}}{2}
\end{array}\right) .
$$

If $\mu \simeq M_{1}$, the Bino and $\tilde{H}_{1}$ are mixed with an angle $\theta_{+}$, but $\tilde{H}_{2}$ is nearly a pure state. If $\mu \simeq-M_{1}$, the mixing occurs between $\tilde{B}$ and $\tilde{H}_{2}$ with mixing angle $\theta_{-}$, while $\tilde{H}_{1}$ is nearly a pure state. However notice that if $\tan \beta=1, \tilde{H}_{2}$ becomes an exact mass eigenstate (to all orders in $M_{2}$ ) which never mixes with $\tilde{B}$. Indeed, $\theta_{-}$vanishes when $\tan \beta=1$. At large $\tan \beta$, the sign of $\mu$ is unphysical and the two cases $\mu \simeq \pm M_{1}$ give identical results.

When $\left|\mu \pm M_{1}\right|<\left(s_{\beta} \mp c_{\beta}\right) s_{W} M_{Z} / \sqrt{2}$, eqs. (91)-(10) are no longer valid. In this case the $\tilde{B}-\tilde{H}_{1}$ mixing angle (for $\mu$ and $M_{1}$ with same sign) or the $\tilde{B}-\tilde{H}_{2}$ mixing angle (for $\mu$ and $M_{1}$ with opposite sign) become maximal. The mass eigenvalues become

$$
\begin{gathered}
M_{1}+\frac{\left(s_{\beta} \pm c_{\beta}\right)}{\sqrt{2}} s_{W} M_{Z}+\left(1 \mp s_{2 \beta}\right) \frac{s_{W}^{2} M_{Z}^{2}}{8 M_{1}}, \\
M_{1}-\frac{\left(s_{\beta} \pm c_{\beta}\right)}{\sqrt{2}} s_{W} M_{Z}+\left(1 \mp s_{2 \beta}\right) \frac{s_{W}^{2} M_{Z}^{2}}{8 M_{1}} \\
-M_{1}-\left(1 \mp s_{2 \beta}\right) \frac{s_{W}^{2} M_{Z}^{2}}{4 M_{1}} .
\end{gathered}
$$

Notice that two neutralino masses are split by terms $\mathcal{O}\left(M_{Z}\right)$.

The relic abundance of the tempered $\tilde{B} / \tilde{W}$ is determined by coannihilation among the 3 neutral states and one chargino with mass approximately equal to $\mu$. Coannihilation of different species $\chi_{i}$ is effective as long as the scattering processes $\chi_{i} q \leftrightarrow \chi_{j} q^{\prime}$ (where $q$ and $q^{\prime}$ are relativistic particles) are in equilibrium. This requires $\Gamma_{S}>H$, where $H$ is the Hubble constant today, the scattering rate is $\Gamma_{S} \simeq T^{3} \theta^{2} / M_{\chi}^{2}$ and $M_{\chi}, \theta$ are the typical $\chi$ mass and mixing angle. Therefore our discussion is valid as long as $\theta \gg\left(x_{f} \sqrt{g_{*}} M_{\chi} / M_{\mathrm{Pl}}\right)^{1 / 2}$.

An analytic approximation of the relic abundance of well-tempered neutralinos is described in appendix A, in the limit of small mixing angle. The numerical result for the parameters required to obtain the correct value of $\Omega_{\mathrm{DM}} h^{2}$, using the DARKSUSY package [28], is shown in fig. 2. With about $10 \%$ degeneracy between $\mu$ and $M_{1}$, one can overcome the supersymmetric dark-matter impasse and reproduce the correct relic density for soft terms in the few-hundred $\mathrm{GeV}$ range. We can also have a dark-matter neutralino with $|\mu|<M_{1}$, 


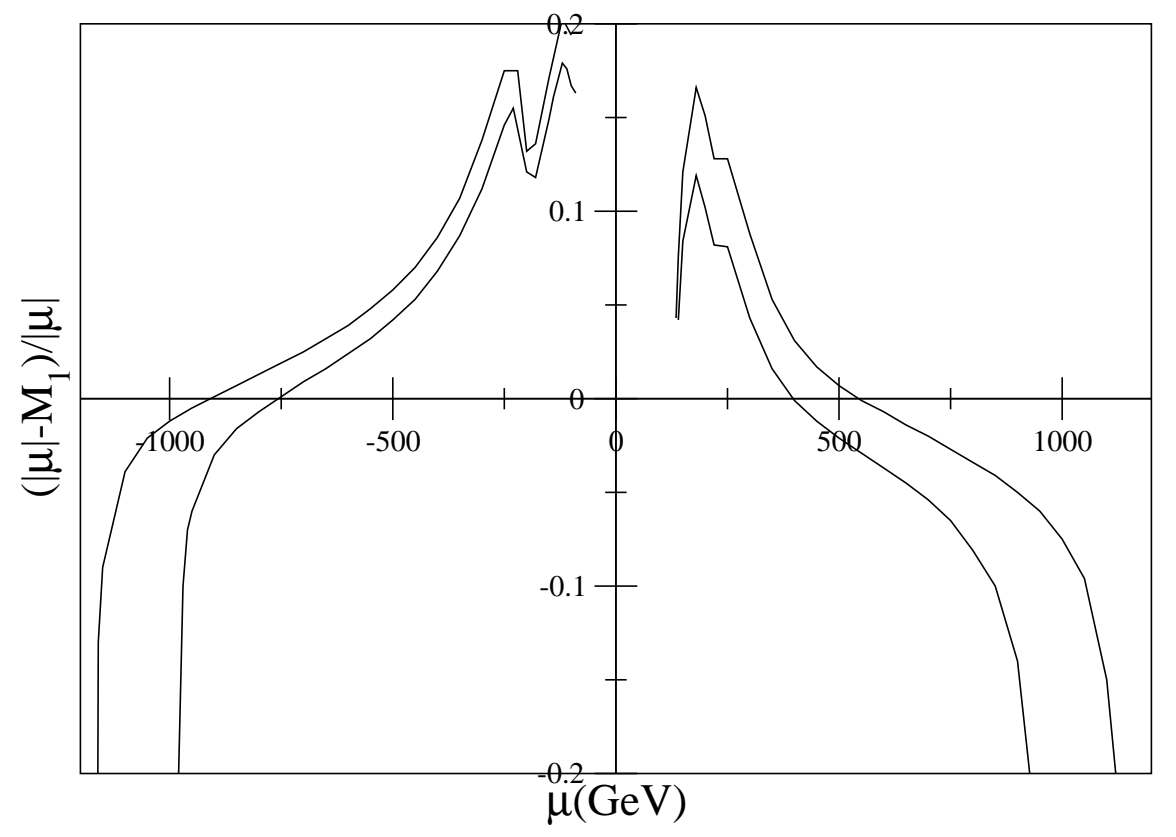

Figure 2: The parameters of the well-tempered $\tilde{B} / \tilde{H}$ consistent with the dark-matter constraint within $2 \sigma$. We have taken $\tan \beta=2, m_{H}=115 \mathrm{GeV}$, and heavy supersymmetric scalars, and chosen the convention $M_{1}>0$. We have considered only $|\mu|>100 \mathrm{GeV}$, to satisfy the experimental limit on chargino masses.

and $\mu$ substantially smaller than $1 \mathrm{TeV}$. This requires a large mixing angle with the $\tilde{B}$ to reduce the Higgsino annihilation cross section. This is why in fig. 2, the region with $|\mu|<M_{1}$ is more prominent in the positive- $\mu$ branch since, in the negative- $\mu$ branch, $\theta_{-}$is suppressed by the moderate value of $\tan \beta$. The dip of the negative- $\mu$ branch in fig. 2 corresponds to the threshold for $t \bar{t}$ production $\left(m_{\chi^{0}}>m_{t}\right)$, below which a higher degree of degeneracy is needed to obtain the same value of $\Omega_{\mathrm{DM}}$. For small $\mu$, the behaviour of the curves with positive and negative $\mu$ is quite distict. Indeed, when eqs. (12)-(14) are approximately valid, the mass splitting in the positive- $\mu$ branch is $\left(t_{\beta}+1\right) /\left(t_{\beta}-1\right)$ times larger than in the negative branch. For $\tan \beta=2$ (the case shown in fig. 2) the mass splitting for moderate and positive $\mu$ becomes large enough to make coannihilation irrelevant. Then the dominant annihilation channel is into Higgs and longitudinal gauge bosons. This explains why the $t \bar{t}$ threshold is less important for $\mu>0$, while the Higgs threshold has a dramatic effect.

When $\mu$ is very close to its minimum value determined by the limit on chargino masses, we cannot really talk about a well-tempered neutralino, since $\mu$ and $M_{1}$ are comparable to $M_{Z}$. In this case, the neutralino becomes a natural mixture of current eigenstates. The supersymmetric dark-matter impasse can be resolved in this region, where $\mu \sim M_{1} \sim M_{Z}$. However, experimental bounds on chargino masses strongly limit the size of this region. 
The well-tempered $\tilde{B} / \tilde{H}$ is favourable for dark-matter detection [25]. The spin-independent neutralino-proton cross section mediated by the Higgs boson is given by [29]

$$
\begin{gathered}
\sigma_{p}=\left(\frac{115 \mathrm{GeV}}{m_{H}}\right)^{4} \gamma^{2} 5.4 \times 10^{-43} \mathrm{~cm}^{2} \\
\gamma=\frac{t_{W}}{\sqrt{2}} N_{11}\left[N_{12}\left(c_{\beta}+s_{\beta}\right)+N_{13}\left(c_{\beta}-s_{\beta}\right)\right],
\end{gathered}
$$

where $N$ is the matrix that diagonalizes $\mathcal{M}$. When the mixing is moderate and eqs. (91)-(10) are valid, we find

$$
\gamma \simeq t_{W}^{2} M_{W} \frac{M_{1}+\mu s_{2 \beta}}{\mu^{2}-M_{1}^{2}}
$$

At large $\tan \beta$, the detection rate is independent of $\tan \beta$ and on the sign of $\mu$. For small $\tan \beta$, $\gamma \simeq t_{W}^{2} M_{W} /\left(\mu-M_{1}\right)$ is significant for same-sign $M_{1}-\mu\left(\tilde{B}-\tilde{H}_{1}\right.$ mixing $)$, but it is suppressed for opposite sign $\left(\tilde{B}-\tilde{H}_{2}\right.$ mixing). On the other hand, when $\left|\mu \pm M_{1}\right|<\left(s_{\beta} \mp c_{\beta}\right) s_{W} M_{Z} / \sqrt{2}$ and we have maximal mixing, then $\gamma=t_{W}\left(c_{\beta} \pm s_{\beta}\right) /(2 \sqrt{2})$, in the cases of same-sign and opposite-sign, respectivey.

The well-tempered $\tilde{B} / \tilde{H}$ can also be detected through neutrino fluxes from the sun [25], since its coupling to the $Z$ is non-vanishing, whenever the mixing angles $\theta_{ \pm}$are large. It may also lead to observable signals in antimatter cosmic rays from annihilation in the halo [25]. Of course, the larger the coannihilation effect, the smaller the LSP annihilation cross section, and therefore the more suppressed are the indirect dark-matter signals from annihilation in the halo.

\section{The Well-Tempered Bino/Wino}

The well-tempered $\tilde{B} / \tilde{W}$ state is effectively described by the $2 \times 2$ mass matrix

$$
\mathcal{M}=\left(\begin{array}{cc}
M_{1} & 0 \\
0 & M_{2}
\end{array}\right)-s_{2 \beta} \frac{M_{Z}^{2}}{\mu}\left(\begin{array}{cc}
s_{W}^{2} & -s_{W} c_{W} \\
-s_{W} c_{W} & c_{W}^{2}
\end{array}\right)+\mathcal{O}\left(\frac{1}{\mu^{2}}\right)
$$

with $\left|M_{1}\right| \simeq\left|M_{2}\right|$. The general discussion including CP-violating effects is given in appendix $\mathrm{B}$, while here we consider the case of real parameters. Notice that the leading term in the $1 / \mu$ expansion vanishes at large $\tan \beta$. Therefore it is useful to give the expressions of the mass eigenvalues and the $\tilde{B} / \tilde{W}$ mixing angle, including terms $1 / \mu^{2}$. We define

$$
\theta \equiv \frac{s_{2 W} s_{2 \beta} M_{Z}^{2}}{2 \mu \Delta M_{1}}, \quad \delta \equiv \frac{s_{2 W} M_{Z}^{2}}{2 \mu^{2} \Delta}, \quad \Delta \equiv \frac{M_{2}-M_{1}}{M_{1}}
$$


First consider the case in which $M_{2}-M_{1}$ is not too small, such that both $\theta$ and $\delta$ are smaller than one. Then the $\tilde{B} / \tilde{W}$ mixing angle is

$$
-N_{12}=\theta+\left(\frac{1}{t_{W}}-t_{W}\right) \theta^{2}+\delta
$$

where $N$ is the orthogonal matrix that diagonalizes the neutralino mass matrix. Notice that $\theta$ approximately describes the mixing angle for small or moderate $\tan \beta$. For large $\tan \beta$, the mixing angle appears only at $\mathcal{O}\left(1 / \mu^{2}\right)$ and it is given by $\delta$. For $M_{1} \simeq M_{2}$ the mixing angle can become large, while for $M_{1} \simeq-M_{2}$ the mixing angle is negligible. The mass eigenvalues of the neutral and charged states are

$$
\begin{gathered}
m_{\chi_{1}}=M_{1}\left[1-\Delta\left(t_{W} \theta+\theta^{2}+t_{W} \delta\right)\right] \\
m_{\chi_{2}}=M_{1}\left[1+\Delta\left(1-\frac{\theta}{t_{W}}+\theta^{2}-\frac{\delta}{t_{W}}\right)\right] \\
m_{\chi^{+}}=M_{1}\left[1+\Delta\left(1-\frac{\theta}{t_{W}}-\frac{\delta}{t_{W}}\right)\right] .
\end{gathered}
$$

Notice that $m_{\chi^{+}}$and $m_{\chi_{2}}$ are split only by $1 / \mu^{2}$ effects, and therefore the two states are nearly mass degenerate.

When $M_{1}-M_{2}$ is so small that $\theta$ or $\delta$ become larger than unity, the photino is a mass eigenstate and the $\tilde{B} / \tilde{W}$ mixing angle is approximately equal to $\theta_{W}$. The mass eigenvalues are

$$
\begin{gathered}
m_{\chi_{1}}=M_{1} \\
m_{\chi_{2}}=M_{1}\left(1-\frac{s_{2 \beta} M_{Z}^{2}}{\mu M_{1}}-\frac{M_{Z}^{2}}{\mu^{2}}\right) \\
m_{\chi^{+}}=M_{1}\left(1-\frac{s_{2 \beta} M_{W}^{2}}{\mu M_{1}}+\frac{M_{W}^{2}}{\mu^{2}}\right) .
\end{gathered}
$$

In this case, all states are split at $\mathcal{O}(1 / \mu)$, unless $\tan \beta$ is very large.

The relic abundance of the well-tempered $\tilde{B} / \tilde{W}$ is determined in terms of the two masses $M_{1,2}$ and the mixing angle. An analytic approximation is presented in appendix A, in both cases of non-vanishing and vanishing mixing angle. The numerical result, using DARKSUSY [28, is shown in fig. 3]. For a degeneracy between $M_{1}$ and $M_{2}$ of about $10 \%$, darkmatter neutralinos can be brought within the mass range of few hundreds GeV. For larger mixing angles, the degree of degeneracy can be reduced. Sizable mixing angles cannot be obtained in the case in which $M_{1}$ and $M_{2}$ have opposite sign, but they occur for generic phases between $M_{1}$ and $M_{2}$, as discussed in appendix B.

\subsection{Connection with High-Energy Soft Terms}

The relation $\left|M_{1}\right| \simeq\left|M_{2}\right|$, necessary for well-tempered $\tilde{B} / \tilde{W}$, has to hold at the weak scale. At first sight, this appears inconsistent with either gaugino mass unification or anomaly me- 


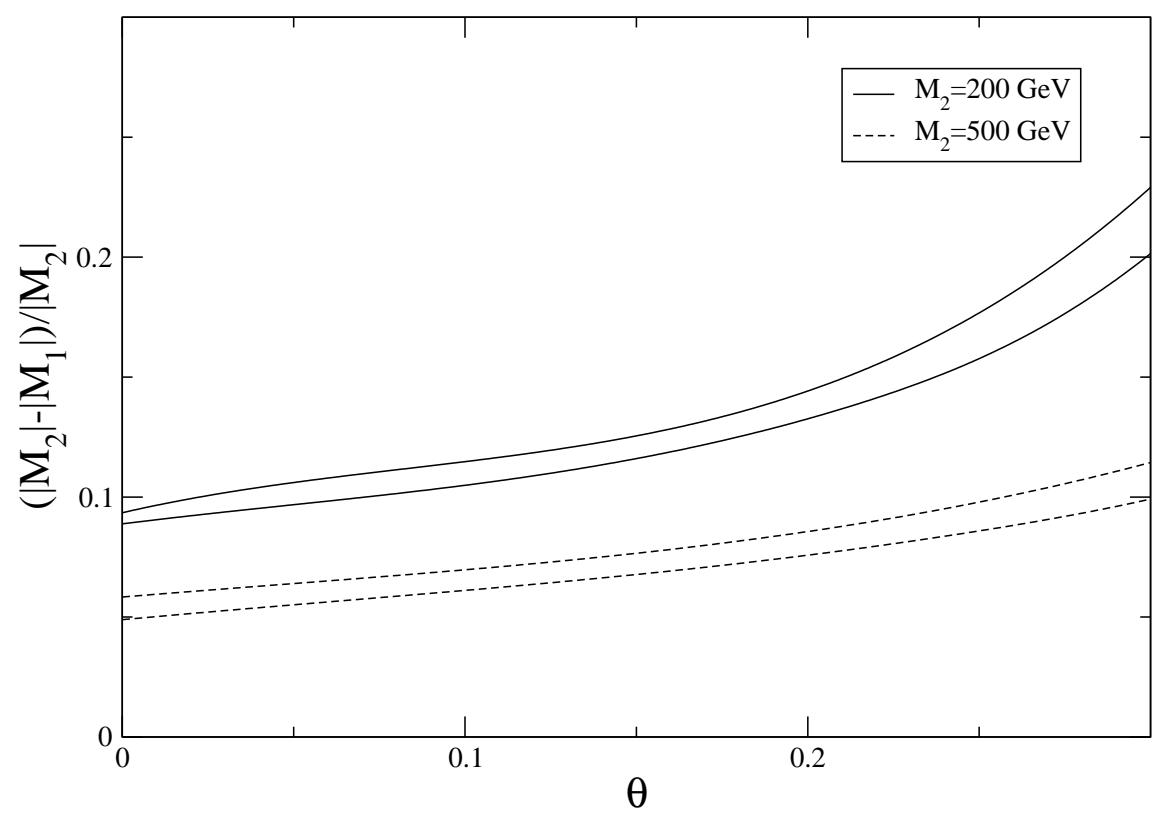

Figure 3: The bands show the parameters of the well-tempered $\tilde{B} / \tilde{W}$ consistent with the dark-matter constraint within $2 \sigma$. We have taken heavy supersymmetric scalars.

diation. However, this is not the case if $\mu$ is much larger than $M_{1,2}$. Indeed, renormalization effects below the scale of the heavy Higgs doublet mass $\left(m_{A}\right)$ generate additive contributions to the gaugino masses proportional to $\mu$. Let us consider the one-loop corrected gaugino masses, including terms enhanced by a logarithm or proportional to $\mu$ [30]

$$
\begin{gathered}
M_{1}=M_{1}\left(m_{A}^{2}\right)\left[1+\frac{\alpha}{8 \pi c_{W}^{2}}\left(11 \log \frac{\tilde{m}_{q}^{2}}{m_{A}^{2}}+9 \log \frac{\tilde{m}_{e}^{2}}{m_{A}^{2}}+\log \frac{\mu^{2}}{m_{A}^{2}}\right)\right] \\
+\frac{\alpha}{8 \pi c_{W}^{2}} \mu s_{2 \beta} f\left(\frac{\mu^{2}}{m_{A}^{2}}\right) \\
M_{2}=M_{2}\left(m_{A}^{2}\right)\left[1+\frac{\alpha}{8 \pi s_{W}^{2}}\left(9 \log \frac{\tilde{m}_{q}^{2}}{m_{A}^{2}}+3 \log \frac{\tilde{m}_{e}^{2}}{m_{A}^{2}}+\log \frac{\mu^{2}}{m_{A}^{2}}-12 \log \frac{M_{2}^{2}}{m_{A}^{2}}\right)\right] \\
+\frac{\alpha}{8 \pi s_{W}^{2}} \mu s_{2 \beta} f\left(\frac{\mu^{2}}{m_{A}^{2}}\right) \\
f(x)=\frac{2 \log x}{1-x},
\end{gathered}
$$

where all coefficients on the right-hand sides of eqs. (23)-(24) have to be evaluated at the scale $m_{A}$. The gaugino masses at the scale $m_{A}$ are obtained by evolving high-energy boundary conditions using renormalization-group flow. For gaugino-mass unification and for anomaly mediation, respectively, we have

$$
\begin{aligned}
& M_{1}\left(m_{A}\right)=\frac{5 \alpha\left(m_{A}\right)}{3 c_{W}^{2} \alpha_{\mathrm{GUT}}} M_{G}, M_{2}\left(m_{A}\right)=\frac{\alpha\left(m_{A}\right)}{s_{W}^{2} \alpha_{\mathrm{GUT}}} M_{G} \quad \text { (gaugino unif.) } \\
& M_{1}\left(m_{A}\right)=\frac{11 \alpha\left(m_{A}\right)}{4 \pi c_{W}^{2}} m_{3 / 2}, M_{2}\left(m_{A}\right)=\frac{\alpha\left(m_{A}\right)}{4 \pi s_{W}^{2}} m_{3 / 2} \quad \text { (anomaly med.) }
\end{aligned}
$$




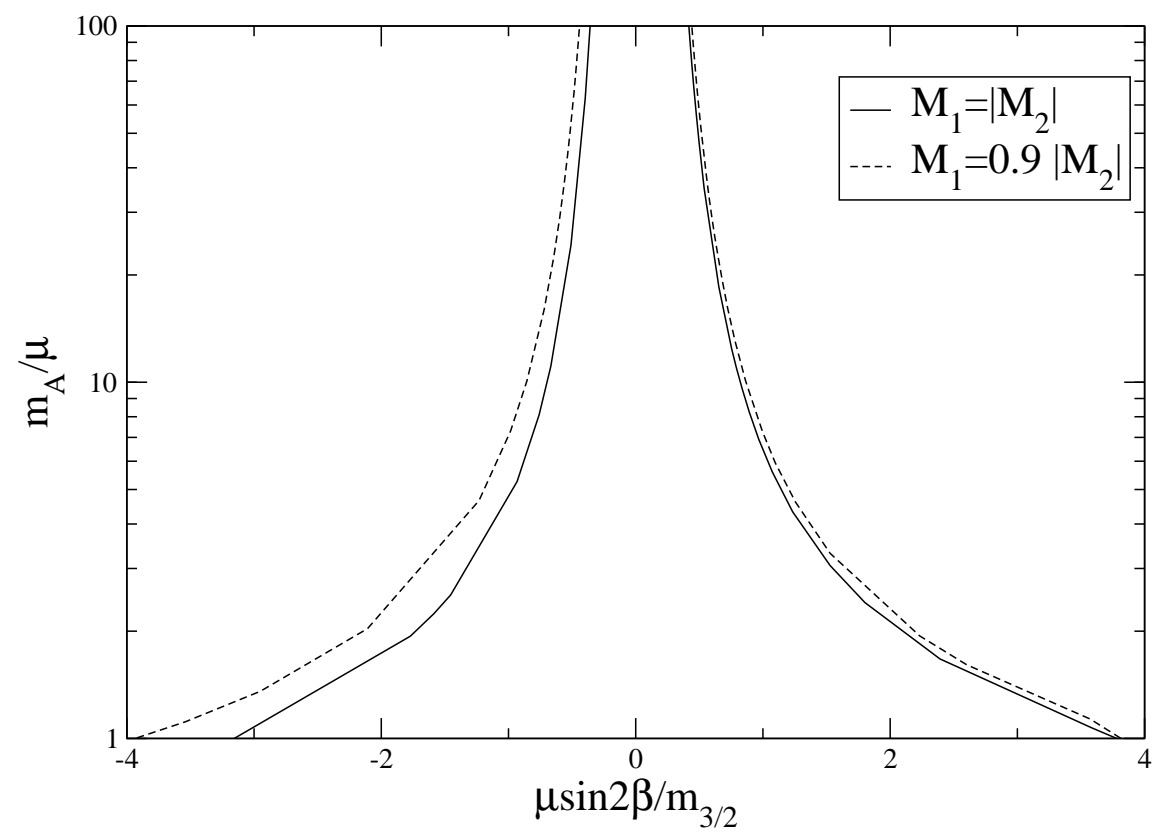

Figure 4: The relation between $\mu, m_{A}$ and $m_{3 / 2}$ necessary to obtain a well-tempered $\tilde{B} / \tilde{W}$ with $0<\left(\left|M_{2}\right|-\left|M_{1}\right|\right) /\left|M_{2}\right|<0.1$, assuming gaugino masses from anomaly mediation.

where $M_{G}$ is the common gaugino mass at the unification scale and $m_{3 / 2}$ is the gravitino mass.

In our numerical analysis, we have actually resummed the logarithms using the renormalization-group equations for Split Supersymmetry, but the one-loop results in eqs. (23)-(24) are useful for our discussion because they explicitly exhibit an essential feature. The quantum corrections proportional to $\mu$ can significantly modify the high-energy gaugino-mass boundary condition (for $\mu \gg M_{1,2}$ ) and they can realize the well-tempered mass relation. In the case of gaugino-mass unification, the relation $M_{1}=M_{2}$ is obtained for $\mu s_{2 \beta} / M_{G}=49$ (for $m_{A} / \mu=10$ ) or 25 (for $m_{A} / \mu=100$ ); the relation $M_{1}=-M_{2}$ is obtained for $\mu s_{2 \beta} / M_{G}=81$ (for $m_{A} / \mu=10$ ) or 40 (for $m_{A} / \mu=100$ ). This requires a certain hierarchy between soft terms, for which we have no good theoretical justification.

More interesting is the case of anomaly mediation because, as shown in fig. 4, the $\tilde{B} / \tilde{W}$ well-tempered relation is obtained for values of $\mu$ of the order of the gravitino mass $m_{3 / 2}$. As we will show in the next section, this can be naturally obtained in simple models of Split Supersymmetry. The two branches of solutions shown in fig. 4 correspond to the case in which $M_{1}$ and $M_{2}$ have equal sign (negative $\mu$ ) and opposite sign (positive $\mu$ ). 


\section{The Simplest Model of Split Supersymmetry}

Here we want to discuss one of the simplest construction of Split Supersymmetry and also show that it can lead to a well-tempered $\tilde{B} / \tilde{W}$. Let us consider the case in which supersymmetry is broken by an auxiliary field in the combination $F^{\dagger} F=M_{S}^{4} \theta^{2} \bar{\theta}^{2}$, generating through direct mediation soft masses for dimension-2 operators: squark and slepton square masses and the $B_{\mu}$ parameter, of the order of $\tilde{m}^{2}=M_{S}^{4} / M^{2}$. We are interested in a mediation scale $M$ in the range $M_{\mathrm{GUT}}<M<M_{\mathrm{Pl}}$, where $M_{\mathrm{Pl}}$ is the reduced Planck mass, so that the new dynamics cannot affect gauge-coupling unification. If the supersymmetry-breaking sector contains no singlets and the breaking is mainly along the $D$-component $F^{\dagger} F$, the direct mediation of the dimension-3 soft-term operators (gaugino masses, $A$ and $\mu$ terms) is suppressed [17. Anomaly mediation then gives the leading contribution. The gaugino masses are generated by the coupling of the chiral compensator $\Phi$ to the gauge kinetic term and are given by $M_{g}(Q)=(d \log g / d \log Q) m_{3 / 2}$ [18, 19].

There are two couplings of the chiral compensator to the Higgs superfields that can generate a $\mu$ term:

$$
\mathcal{L}=\lambda \int d^{4} \theta \Phi^{\dagger} \Phi H_{u} H_{d}+\left(\rho \int d^{2} \theta \Phi^{3} H_{u} H_{d}+\text { h.c. }\right) .
$$

Performing a field rescaling $\Phi H_{u, d} \rightarrow H_{u, d}$, the Lagrangian becomes

$$
\mathcal{L}=\lambda \int d^{4} \theta \frac{\Phi^{\dagger}}{\Phi} H_{u} H_{d}+\left(\rho \int d^{2} \theta \Phi H_{u} H_{d}+\text { h.c. }\right) .
$$

Since $\Phi=1+\theta^{2} m_{3 / 2}$, we obtain the following contributions to the $\mu$ and $B_{\mu}$ terms, respectively,

$$
\mu=\lambda m_{3 / 2}+\rho, \quad B_{\mu}=\lambda m_{3 / 2}^{2}-\rho m_{3 / 2}
$$

The parameter $\rho$ is dimensionful and it is a manifestation of the $\mu$ problem, since it originates from supersymmetric-invariant dynamics and its value is not, in principle, related to $m_{3 / 2}$. On the other hand, the coupling $\lambda$ corresponds to the natural solution of the $\mu$-problem in supergravity [31].

If $\rho$ vanishes and the contribution to $B_{\mu}$ from direct coupling to the supersymmetrybreaking sector is suppressed, then from eq. (30) we find the relation $B_{\mu}=\mu m_{3 / 2}$. Minimization of the Higgs potential gives $s_{2 \beta}=2 B_{\mu} / m_{A}^{2}$, where $m_{A}$ is the mass of the heavy Higgs doublet, which is of the order of $\tilde{m}$. Therefore, in this case, an acceptable value of $\tan \beta$ requires $\tilde{m} \sim m_{3 / 2}$ and the scale of supersymmetry-breaking mediation $M$ is near the Planck scale. Also notice that in this case we find

$$
\frac{\mu s_{2 \beta}}{m_{3 / 2}}=\frac{2 \mu^{2}}{m_{A}^{2}} .
$$


This relation corresponds to the model considered in ref. [19]. It is compatible with a welltempered $\tilde{B} / \tilde{W}$ (with opposite signs for $M_{1}$ and $M_{2}$ ) when $m_{A}=0.57 \mu$. Through eq. (31) this predicts $\mu s_{2 \beta}=6.1 m_{3 / 2}$. Also notice that, if $\rho$ were the dominant source for $B_{\mu}$, we would obtain $B_{\mu}=-\mu m_{3 / 2}$, which leads to the relation $\mu s_{2 \beta} / m_{3 / 2}=-2 \mu^{2} / m_{A}^{2}$. This is compatible with a well-tempered $\tilde{B} / \tilde{W}$ (with equal signs for $M_{1}$ and $M_{2}$ ) when $m_{A}=0.55|\mu|$, leading to $\mu s_{2 \beta}=-6.6 m_{3 / 2}$. In general, however, the direct mediation contribution to $B_{\mu}$ is significant and the relation in eq. (31) will not be strictly exact.

To summarize, the Simplest Model of Split Supersymmetry (SMS) is characterized by: (i) Squark, slepton masses and $B_{\mu}$ of typical size $\tilde{m}=k m_{3 / 2}$, with $1 \lesssim k \lesssim M_{\mathrm{Pl}} / M_{\mathrm{GUT}}$; (ii) $\mu$ parameter of typical size $\mu \sim m_{3 / 2}$; (iii) Gaugino masses given by the anomaly-mediation relation $\left(M_{\tilde{g}} \sim m_{3 / 2} g^{2} / 16 \pi^{2}\right)$, with large corrections to $M_{1,2}$ proportional to $\mu s_{2 \beta}$ given by eqs. (23) -(24). Therefore, the SMS is described by 4 parameters $\left(\tilde{m}, m_{3 / 2}, \mu, \tan \beta\right)$, with typical mass ratios, as specified above. It is, in our view, the most straightforward construction of a model with Split Supersymmetry. It is a variation of the model first considered in ref. [19], and then revived in ref. [32].

There is another limit, considered in ref. [15], where the direct coupling of the Higgses to the supersymmetry-breaking sector is not suppressed, so that $B_{\mu} \epsilon \sim \tilde{m}^{2}$ where $\epsilon$ is a parameter characterizing the size of PQ breaking. This leads to $\tan \beta \sim 1 / \epsilon$, and also $\mu \sim \epsilon m_{3 / 2} \sim m_{3 / 2} / \tan \beta$. In this case it is possible to have Higgsinos near the TeV scale with large $\tan \beta$.

The SMS is minimal in the sense that essentially any model of supersymmetry breaking, as long as it does not have singlets to directly mediate gaugino masses, will produce this spectrum without additional special structure to "sequester" the scalar masses from supersymmetry breaking. There are exceptions where, for good physical reasons, the anomaly mediated contributions are suppressed as described in ref. [15] and in detail in ref. [17]. If supersymmetry is broken already in global limit, $F_{\Phi}=m_{3 / 2}$ and the anomaly mediated contribution is there, while if supersymmetry is restored as gravity is decoupled, $F_{\phi} \rightarrow 0$ and anomaly mediation is shut off. But there are certainly wide classes of models of both types. SMS makes the scalars heavy enough to eliminate all the usual problems of low-energy supersymmetry.

The requirement of an appropriate thermal dark-matter density can be satisfied when the LSP Wino has a mass of about 2.5 TeV, or with light Higgsinos near $1 \mathrm{TeV}^{1}$. In either of

\footnotetext{
${ }^{1}$ Note that with the gravitino near $100 \mathrm{TeV}$, the decay of the gravitino can dilute the thermal relic abundance, so we must assume a low enough reheating temperature for this not to happen. An alternative cosmological history in this model is examined in 33.
} 
these cases, no observable signals can be expected at the LHC. More appealing is the case in which the SMS leads to a well-tempered $\tilde{B} / \tilde{W}$. The necessary relation among $\mu, \tilde{m}\left(=m_{A}\right)$, $m_{3 / 2}$, and $\tan \beta$ is illustrated in fig. 4. It is interesting to notice that the SMS expectations $\mu / m_{3 / 2}=\mathcal{O}(1)$ and $\tilde{m} / \mu=\mathcal{O}(k)$ are exactly what is needed to obtain a well-tempered $\tilde{B} / \tilde{W}$, whenever $s_{2 \beta}$ is not too small (i.e. $\tan \beta$ is not too large). Notice that for complex soft parameters, the values of $|\mu| s_{2 \beta} / m_{3 / 2}$ that lead to a well-tempered $\tilde{B} / \tilde{W}$ vary, as we change the relative phase $\varphi$ between $\mu$ and $m_{3 / 2}$, in the interval $\left|\mu_{+}\right| \leq|\mu| \leq\left|\mu_{-}\right|$, where $\mu_{ \pm}$ are the solutions in the positive and negative branches shown in fig. 4.

Once the well-tempered condition is verified, and the parameters satisfy the relation $\left|M_{1}\right|=\left|M_{2}\right|$, then the ratio between the gaugino and gravitino mass is fixed, and it is independent of the value of $\mu$, up to small logarithmic effects, depending only on the phase $\varphi$. We find $m_{3 / 2} /\left|M_{2}\right|=a_{1}(\varphi)$, where $a_{1}(\pi) \simeq 80$ (negative- $\mu$ branch) and $a_{1}(0) \simeq 150$ (positive- $\mu$ branch), and intermediate values between 80 and 150 are obtained with a generic phase $\varphi$.

The well-tempered condition eliminates one parameter, and therefore the SMS can be described in terms of: (i) an overall mass scale, which we take to be the gaugino mass $\left|M_{2}\right|$; (ii) the mass ratio $\tilde{m} / M_{2}$, which describes the degree of mass hierarchy or, in other words, the amount of Split Supersymmetry; (iii) $\tan \beta$, which cannot be too large, or else the radiative contribution to gaugino masses proportional to $\mu$ is suppressed; (iv) the relative sign between $\mu$ and $m_{3 / 2}$ (or more generally, for complex terms, the phase $\varphi$ ). The theory also selects an allowed interval for the ratio $\tilde{m} /\left|M_{2}\right|$ :

$$
\frac{a_{2}(\varphi)}{s_{2 \beta}}<\frac{\tilde{m}}{\left|M_{2}\right|}<a_{1}(\varphi) \frac{M_{\mathrm{Pl}}}{M_{\mathrm{GUT}}}
$$

where $a_{2}(\pi) \simeq 260($ negative $\mu)$ and $a_{2}(0) \simeq 570($ positive $\mu)$.

The lower bound on $\tilde{m} /\left|M_{2}\right|$ is obtained from the requirement that the well-tempered condition can be achieved. The contribution to gaugino masses $\mu / \tilde{m} f\left(\mu^{2} / \tilde{m}^{2}\right)$ in eqs. (23) and (24) has a minimum for $\mu=\tilde{m}$, which determines the lower limit in eq. (32). The upper bound on $\tilde{m} /\left|M_{2}\right|$ is obtained from the ratio $m_{3 / 2} /\left|M_{2}\right|$, using $\tilde{m} /\left|M_{2}\right|=a_{1}(\varphi) k$ and $k \lesssim M_{\mathrm{Pl}} / M_{\mathrm{GUT}}$. Therefore, from eq. (32) we infer that the SMS can vary from a mild to a more accentuated version of Split Supersymmetry, with a mass hierarchy between supersymmetric scalars and fermions which is, however, never extreme.

The mass of the gluino in the SMS is also fixed in terms of the other parameters and in fig. [5 we show the ratio between the gluino pole mass and the gaugino mass as a function of $\tilde{m}$. In the negative- $\mu$ branch, the ratio $M_{\tilde{g}} / M_{2}$ is sufficiently small to allow for a large 


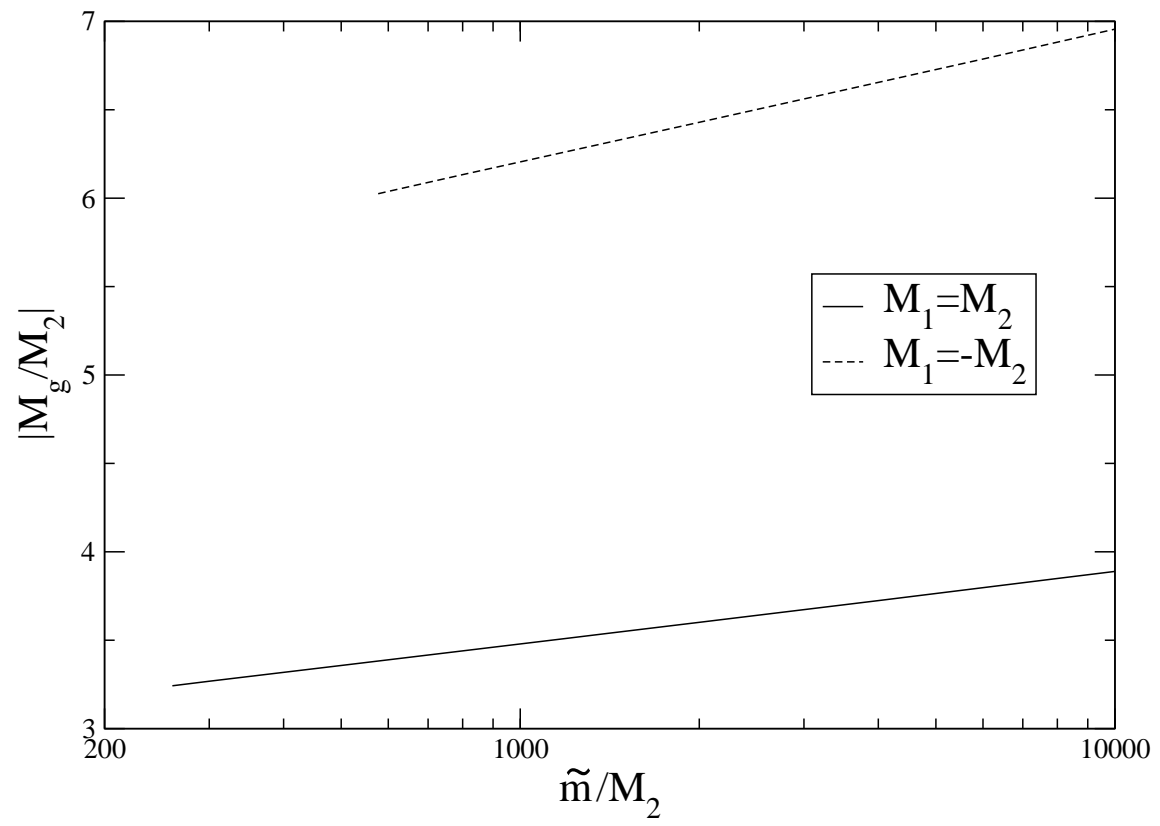

Figure 5: The ratio $M_{\tilde{g}} /\left|M_{2}\right|$ between the gluino pole mass and the gaugino mass as a function of $\tilde{m} /\left|M_{2}\right|$ for $\tan \beta=1$, assuming the well-tempered $\tilde{B} / \tilde{W}$ relation in the negative- $\mu$ branch $\left(M_{1}=M_{2}\right)$ and the positive- $\mu$ branch $\left(M_{1}=-M_{2}\right)$. The curves start from the minimum value of $\tilde{m} /\left|M_{2}\right|$ determined by eq. (32).

mass range where both the gluino and neutralinos/charginos are within the kinematic reach of LHC. The situation is less favourable in the positive- $\mu$ branch, where $M_{\tilde{g}} / M_{2}$ is almost twice as large. Nevertheless, this ratio is somehow smaller than in the case of pure anomaly mediation, where $M_{\tilde{g}} / M_{2}=7.1$ [21].

The gluino lifetime is 34

$$
\tau_{\tilde{g}}=\left(\frac{\tilde{m}}{10^{5} \mathrm{GeV}}\right)^{4}\left(\frac{\mathrm{TeV}}{M_{\tilde{g}}}\right)^{5} 7 \times 10^{-16} \mathrm{sec} .
$$

Before decaying, the gluino will travel a mean distance $d \sim \tau_{\tilde{g}} p / M_{\tilde{g}}$, where $p$ is the gluino momentum. Therefore, from eq. (33), we infer that the SMS gluino always decays inside the detector since, as previously discussed, $\tilde{m} / M_{2}$ is expected to lie between $10^{2}$ and $10^{4}$. A vertex displacement can be observed by LHC detectors if $\tau_{\tilde{g}} \gtrsim 10^{-12}$ sec, which implies

$$
\tilde{m} \gtrsim\left(\frac{M_{\tilde{g}}}{\mathrm{TeV}}\right)^{5 / 4} 600 \mathrm{TeV} .
$$

Therefore, in a range of SMS parameters, measurements at the LHC can determine the value of $\tilde{m}$. However, it will be more difficult to distinguish the SMS with $\tilde{m}$ below the value in eq. (34) from a more conventional supersymmetry with scalars barely escaping searches at the LHC. One possibility to disantangle the two scenarios is offered by the 
search for gluinonium [35]. In the limit of heavy scalars, the gluino decay process is slower than the gluino annihilation inside the gluinonium bound state. Particularly interesting is the ${ }^{3} S_{1}\left(\mathbf{8}_{A}\right)$ gluinonium state, which cannot decay into two gluons (a final state with large QCD background from dijets), but only into quark-antiquark pairs. Bottom and/or top identification can help reducing the background. The ${ }^{3} S_{1}\left(\mathbf{8}_{A}\right)$ decay width into massless quarks is approximately [35]

$$
\Gamma=\frac{81}{32} \alpha_{s}\left(M_{\tilde{g}}\right)^{5} M_{\tilde{g}}
$$

with about $1 / 6$ branching ratio in each quark channel. If $\tilde{m}$ is few times bigger than $M_{\tilde{g}}$, then $\Gamma$ is larger than $\tau_{\tilde{g}}^{-1}$, and the gluinonium annihilates before its gluino components can decay. Observation of gluinonium could provide an indication to discriminate between the SMS and more conventional supersymmetry, with squarks relatively close in mass to the gluino.

The search for neutralinos and charginos at the LHC will be challenging. These particles will be produced by Drell-Yan processes or via gluino decay. Signals are very sensitive on the mass difference between the states [26] and they can be hard to detect when the decay products become soft. More studies are needed on the detection of quasi-degenerate neutralinos and charginos.

For complex soft parameters, the SMS will be well tested by present and future experimental searches for electric dipole moments [36]. Notice that the low-energy gaugino masses $M_{1,2}$ are given by the sum of two comparable contributions: one proportional to $m_{3 / 2}$ and one to $\mu$, see eqs. (23) -(24), with a relative phase $\varphi$. Therefore, in contrast to the studied versions of Split Supersymmetry, the two physical CP-violating phases $\arg \left(M_{1,2} \mu\right)$ are different, and can be suppressed if the $\mu$-contribution to gaugino masses is dominant. For $M_{1}$, the pure anomaly-mediation contribution is equal to 0.8 (1.5) times the total value of $M_{1}$ in the negative (positive) $\mu$-branch, and this will not suppress $\arg \left(M_{1} \mu\right)$. However, we expect a certain suppression of $\arg \left(M_{2} \mu\right)$, since the pure anomaly-mediation contribution to $M_{2}$ is equal to $0.3(-0.5)$ times the total, in the negative (positive) $\mu$-branch. The SMS contribution to electric dipole moments is smaller than in Split Supersymmetry, because Higgsinos are heavier, but it can still be detected in future experiments. If $\tilde{m}$ is not much larger than $m_{3 / 2}$, then the leading contribution to electric dipole moments in the SMS comes from one-loop diagrams mediated by scalar particles. For large phases, the effect can be detected in future experiments. 


\section{Conclusions}

We have argued that, after the unsuccessful searches at LEP, it is no longer justified to claim that the correct value of $\Omega_{\mathrm{DM}}$ is a natural prediction of low-energy supersymmetry. Once the experimental constraints are satisfied, low-energy supersymmetry typically leads to a too large $\left(\tilde{B}\right.$ LSP) or too small $\left(\tilde{H}\right.$ or $\tilde{W}$ LSP) value of $\Omega_{\mathrm{DM}}$. Notwithstanding, the minimal supersymmetric model can successfully reproduce the correct value of $\Omega_{\mathrm{DM}}$. However, apart from a small region with sleptons or with $\mu$ and $M_{1}$ very close to their present experimental limits, the prediction for $\Omega_{\mathrm{DM}}$ requires a critical choice of parameters. It is critical in the sense that it is highly sensitive on small parameter variations. In particular, when we rely on coannihilation, $\Omega_{\mathrm{DM}}$ exponentially depends on the difference between two a priori unrelated soft parameters. Of course this is not a problem of consistency, but only a conceptual difficulty completely analogous to the fine-tuning problem of electroweak-symmetry breaking. In low-energy supersymmetry, the correct value of $M_{Z}$ can be reproduced by the soft terms, but only at the price of critical choices of the parameters (i.e. with few-percent sensitivity on small variations). We view it as disturbing that two of the most important conceptual achievements of low-energy supersymmetry - electroweak breaking and dark matter - both require special conspiracies on the values of the underlying soft parameters. Of course, from a more optimistic point of view, one can view both problems as the result of a fortuitous and unlucky occurrence that has determined values of the soft terms highly untypical of the acceptable parameter space. After all, it is admittedly remarkable and non-trivial that both values of $M_{Z}$ and $\Omega_{\mathrm{DM}}$ can be successfully computed in terms of soft-breaking parameters.

If we insist that the dark matter originates from a supersymmetric thermal relic, free from any dependence on the cosmological evolution above the freeze-out temperature $T_{f}$, then we have to accept that the soft terms approximately satisfy some critical condition. We have argued that the case of the well-tempered neutralino is one of the most plausible of these choices. A well-tempered neutralino corresponds to the boundary between a $\tilde{B}$ LSP and a $\tilde{H}$ or $\tilde{W}$ LSP, where $\Omega_{\mathrm{DM}}$ rapidly varies from being too large to being too small. We have studied the properties of well-tempered neutralinos, also considering the case of complex soft terms. Since in the general parameter space of low-energy supersymmetry, well-tempered neutralinos are some of the most favourable candidates for dark matter, we believe that there are good motivations for dedicated studies on the detection of quasi-degenerate neutralinos and charginos at the LHC.

We have also shown that the $\tilde{B} / \tilde{W}$ well-tempered condition $\left|M_{1}\right| \simeq\left|M_{2}\right|$ can be satisfied in models with conventional high-energy boundary conditions for the gaugino mass, taking a 
large $\mu$ term. One of the most interesting models of this class is the SMS, discussed in sect. 4, which is the simplest realization of Split Supersymmetry. In the SMS, direct couplings to a hidden sector generate the supersymmetric scalar masses, and anomaly mediation generates the masses for the supersymmetric fermions. The SMS is consistent with a well-tempered $\tilde{B} / \tilde{W}$ and, once the appropriate relation is imposed, we find specific predictions for the mass ratios. It is not an extreme version of Split Supersymmetry, since the ratio between the common scalar masses and the gaugino mass $\tilde{m} / M_{2}$ varies between few $10^{2}$ and about $10^{4}$, see eq. (32). As a function of $\tilde{m} / M_{2}, \tan \beta$ and (for complex parameters) the phase of $\mu$, the mass ratios $\mu / M_{2}$ and $M_{\tilde{g}} / M_{2}$ are determined, see figs. 4 and 5 .

Finally, we want to remark that the supersymmetric dark-matter impasse, discussed in sect. 1, does not immediately apply to Split Supersymmetry, since values of $\mu$ of about $1 \mathrm{TeV}$ or $M_{2}$ of about $2.5 \mathrm{TeV}$ are perfectly acceptable, once we abandon the naturalness criterion. Why then should we expect to have an extra tuning to get well-tempered neutralinos? It is difficult to answer this question without having a more precise notion of what the physical measure of tuning actually is, but we can at least identify a competition between two factors. If we scale up the Wino to $2.5 \mathrm{TeV}$ as the LSP, so there is no tuning for dark matter, we are making the scalars heavier too, which makes electroweak breaking more tuned. If we leave Winos in the hundreds of $\mathrm{GeV}$ range, the scalars are lighter and electroweak breaking is less tuned but there is more tuning to get the dark matter. At any rate, a $2.5 \mathrm{TeV}$ Wino make Split Supersymmetry invisible at the LHC (for conventional gaugino mass relations). Therefore the collider detectability of Split Supersymmetry relies on the existence of welltempered neutralinos.

Of course this conclusion depends on our assumption that the dark matter is entirely composed of the thermal relic. It is possible that the cosmological history is more involved. For instance, in the minimal version of Split Supersymmetry with gravitinos near $\sim 100 \mathrm{TeV}$, for a high reheating temperature the gravitinos are produced with sufficient abundance so that their (safe) decay before nucleosynthesis nonetheless significantly dilutes the LSP abundance. Depending on the presence of other light moduli fields with masses around $\tilde{m}$, the LSPs can be repopulated, and so lighter LSPs can form the dark matter, with a visible gluino at the LHC [33. Alternately, there may be more than one source of dark matter, for instance not just our LSP but also axions. It is further plausible that the dark-matter abundance is "environmentally" selected for [37. In this case, both the initial amplitude of the axion as well as the scale of gaugino masses need to be lowered in order to get a small enough dark-matter abundance, but it is reasonable to expect comparable abundances from each one. This would again mean that we could have a lighter LSP, now providing an $\mathcal{O}(1)$ 
fraction of the DM, but again with a spectrum visible at the LHC.

However, the experimental observation of Split Supersymmetry with a well-tempered neutralino for dark matter would be very striking. Even in the minimal version of Split Supersymmetry with anomaly mediated gaugino masses and scalars in the range between $10^{2}-10^{3} \mathrm{TeV}$, there would be evidence for an enormous tuning for electroweak symmetry breaking of about 1 part in $\sim 10^{6}$. This would already represent a severe blow against naturalness, only further augmented by the additional tuning required for getting the correct dark-matter abundance. Fortunately, the LHC will soon begin to tell us what path Nature has chosen - and whether the weak scale will represent the triumphant return or final downfall of naturalness.

\section{Appendix A}

In this appendix we give the analytic expressions for annihilation and coannihilation processes relevant to the cases of pure states and well-tempered neutralinos, in the limit in which $M_{W}$ is much smaller than the gaugino and Higgsino masses.

The relic abundance is given by

$$
\Omega h^{2}=\frac{688 \pi^{5 / 2} T_{\gamma}^{3}(n+1) x_{f}^{n+1}}{99 \sqrt{5 g_{*}}\left(H_{0} / h\right)^{2} M_{\mathrm{Pl}}^{3} \sigma}=\frac{8.7 \times 10^{-11} \mathrm{GeV}^{-2}(n+1) x_{f}^{n+1}}{\sqrt{g_{*}} \sigma},
$$

where $g_{*}$ is the number of degrees of freedom at freeze-out, $H_{0}$ is the present Hubble constant and $T_{\gamma}$ is the CMB temperature. Here $\sigma$ is related to the thermal-averaged non-relativistic annihilation cross section by

$$
\langle\sigma v\rangle=\sigma x^{-n}, \quad x=\frac{m_{\chi}}{T},
$$

and the freeze-out temperature $T_{f}\left(x_{f}=m_{\chi} / T_{f}\right)$ is

$$
x_{f}=X-\left(n+\frac{1}{2}\right) \log X, \quad X=25+\log \left[(n+1) \frac{g}{\sqrt{g_{*}}} m_{\chi} \sigma 6.4 \times 10^{6} \mathrm{GeV}\right] \text {, }
$$

where $g=2$ are the neutralino degrees of freedom and $m_{\chi}$ is its mass.

When $N$ states with mass $m_{i}$ ( $m_{1}$ being the lightest) and equal number of degrees of freedom participate in the coannihilation process, one defines [6] an effective cross section $\sigma_{e f f}$ in terms of the thermal-averaged cross sections for the individual $\chi_{i} \chi_{j}$ annihilations,

$$
\left\langle\sigma_{i j} v\right\rangle=\sigma_{i j} x^{-n}
$$




$$
\left\langle\sigma_{e f f} v\right\rangle=\frac{\sum_{i, j=1}^{N} w_{i} w_{j} \sigma_{i j} x^{-n}}{\left(\sum_{i=1}^{N} w_{i}\right)^{2}}, \quad w_{i}=\left(\frac{m_{i}}{m_{1}}\right)^{3 / 2} e^{-x\left(\frac{m_{i}}{m_{1}}-1\right)} .
$$

The relic abundance is now given by

$$
\Omega h^{2}=\frac{8.7 \times 10^{-11} \mathrm{GeV}^{-2}}{\sqrt{g_{*}} \int_{x_{f}}^{\infty} d x\left\langle\sigma_{e f f} v\right\rangle x^{-2}} .
$$

If all $N$ states are mass-degenerate, eq. (41) takes the same form as eq. (36) with the replacement $\sigma \rightarrow \sum_{i, j} \sigma_{i j} / N^{2}$

\section{Bino}

The annihilation cross section of a pure $\tilde{B}$ into massless fermions is

$$
\sigma_{\tilde{B} \tilde{B}}=\sum_{f} \frac{g^{4} t_{W}^{4}\left(T_{f}-Q_{f}\right)^{4} r\left(1+r^{2}\right)}{2 \pi m_{\tilde{f}}^{2} x(1+r)^{4}}, \quad x \equiv \frac{M_{1}}{T}, \quad r \equiv \frac{M_{1}^{2}}{m_{\tilde{f}}^{2}},
$$

where $T_{f}$ and $Q_{f}$ are third component of isospin and electric charge of the fermion $f$. The value of $\Omega$ is obtained from eq. (36).

\section{Higgsino}

For the Higgsino we consider 4 coannihilating states with equal mass $\mu$ : $\left(\tilde{H}_{1}, \tilde{H}_{2}, \tilde{H}^{+}, \tilde{H}^{-}\right)$. The neutral states $\tilde{H}_{1,2}$ are defined as in sect. 2. and we treat $\tilde{H}^{+}$and $\tilde{H}^{-}$as independent to have the same number of degrees of freedom for each state. The thermal-averaged cross sections for the individual annihilation processes (assuming $\mu \gg M_{W}$ and heavy supersymmetric scalars) is described by the symmetric matrix $\sigma_{i j}$ with

$$
\begin{gathered}
\sigma_{11}=\sigma_{22}=\frac{g^{4}}{128 \pi \mu^{2}}\left(\frac{3}{2}+t_{W}^{2}+\frac{t_{W}^{4}}{2}\right) \\
\sigma_{12}=\frac{g^{4}}{128 \pi \mu^{2}}\left(\frac{29}{4}+\frac{21}{2} t_{W}^{4}\right) \\
\sigma_{1+}=\sigma_{1-}=\sigma_{2+}=\sigma_{2-}=\frac{g^{4}}{128 \pi \mu^{2}}\left(6+t_{W}^{2}\right) \\
\sigma_{+-}=\frac{g^{4}}{128 \pi \mu^{2}}\left(\frac{29}{4}+t_{W}^{2}+11 t_{W}^{4}\right) \\
\sigma_{++}=\sigma_{--}=\frac{g^{4}}{64 \pi \mu^{2}}
\end{gathered}
$$

The effective cross section $\left\langle\sigma_{e f f} v\right\rangle=\sum_{1, j=1}^{4} \sigma_{i j} / 16$ and the Higgsino relic abundance are given in eqs. (41)-(5) .

\section{Wino}


For the Wino we consider 3 coannihilating states with equal masses $M_{2}:\left(\tilde{W}^{0}, \tilde{W}^{+}, \tilde{W}^{-}\right)$. The symmetric matrix $\sigma_{i j}$ (assuming $M_{2} \gg M_{W}$ and heavy supersymmetric scalars) is given by

$$
\begin{gathered}
\sigma_{00}=\frac{g^{4}}{8 \pi M_{2}^{2}}, \quad \sigma_{0+}=\sigma_{0-}=\frac{7 g^{4}}{32 \pi M_{2}^{2}}, \\
\sigma_{+-}=\frac{9 g^{4}}{32 \pi M_{2}^{2}}, \quad \sigma_{++}=\sigma_{--}=\frac{g^{4}}{16 \pi M_{2}^{2}} .
\end{gathered}
$$

The effective cross section $\left\langle\sigma_{e f f} v\right\rangle=\sum_{1, j=1}^{3} \sigma_{i j} / 9$ and the Wino relic abundance are given in eqs. (6) $-(71)$.

\section{Well-Tempered Neutralino}

Let us consider the case of the tempered $\tilde{B} / \tilde{W}$, where there are 4 coannihilating states: $\left(\tilde{B}, \tilde{W}^{0}, \tilde{W}^{+}, \tilde{W}^{-}\right)$. We compute the leading contributions to the relevant cross sections, in the limit of unbroken electroweak symmetry and heavy supersymmetric scalars. The entries of the matrix $\sigma_{i j}$ relative to Wino states are given by eqs. (48)-(49), while the other entries are given by

$$
\begin{gathered}
\sigma_{\tilde{B} \tilde{B}}=\sigma_{\tilde{B} \tilde{B}}^{(s)}+\sigma_{\tilde{B} \tilde{B}}^{(p)} \\
\sigma_{\tilde{B} \tilde{B}}^{(s)}=\frac{g^{4} \theta^{4} M_{1}^{2}}{2 \pi\left(M_{1}^{2}+M_{2}^{2}\right)^{2}} \\
\sigma_{\tilde{B} \tilde{B}}^{(p)}=\frac{g^{4} t_{W}^{4}}{64 \pi x\left(M_{1}^{2}+\mu^{2}\right)^{4}}\left[M_{1}^{2}\left(3 M_{1}^{4}+2 M_{1}^{2} \mu^{2}+3 \mu^{4}\right)\right. \\
\left.+2 M_{1} \mu\left(M_{1}^{4}+4 M_{1}^{2} \mu^{2}+3 \mu^{4}\right) s_{2 \beta}+3 \mu^{2}\left(M_{1}^{2}+\mu^{2}\right)^{2} s_{2 \beta}^{2}\right] \\
\sigma_{\tilde{B} \tilde{W}^{0}}=\frac{g^{4} \theta^{2}}{8 \pi M_{1}^{2}}\left(1-4 \Delta+\frac{31}{8} \Delta^{2}\right)+\mathcal{O}\left(\Delta^{3}\right) \\
\sigma_{\tilde{B} \tilde{W}^{+}}=\sigma_{\tilde{B} \tilde{W}^{-}}=\frac{g^{4} \theta^{2}}{128 \pi M_{1}^{2}}\left(28-\frac{107}{2} \Delta+\frac{141}{2} \Delta^{2}\right)+\mathcal{O}\left(\Delta^{3}\right) .
\end{gathered}
$$

The $\tilde{B} / \tilde{W}$ mixing angle $\theta$ and the mass-splitting parameter $\Delta$ are defined in eq. (19). $\sigma_{\tilde{B} \tilde{B}}^{(p)}$ does not vanish in the limit $\theta \rightarrow 0$, corresponding to the contribution from the Bino annihilation into Higgs bosons and longitudinal gauge bosons via $t$-channel Higgsino exchange. This process can only occur in $p$-wave, as exhibited by the factor $x=M_{1} / T$ in eq. (52), but it becomes important in the limit of small mixing angle. In eq. (52) we have neglected the lightest Higgs mass and taken the pseudoscalar Higgs mass to be larger than $M_{1,2}$. The value of the relic abundance, determined by eqs. (401)-(41) is in excellent agreement with the results obtained with DARKSUSY [28, in the region of small $\theta$ and quasi-degenerate gauginos. 
It is interesting to consider the limit of vanishing mixing angle $\theta$ (but not exactly zero, in order to allow equilibrium between $\tilde{B}$ and $\tilde{W}$ number densities). The relic density of well-tempered $\tilde{B} / \tilde{W}$ neutralinos (with $\left|M_{1}\right|<\left|M_{2}\right|$ ) is completely determined by the $\tilde{W}$ annihilation processes (neglecting the small $p$-wave annihilation into Higgs fields) and we find

$$
\begin{gathered}
\Omega_{\tilde{B} \tilde{W}} h^{2}=0.13\left(\frac{M_{2}}{2.5 \mathrm{TeV}}\right)^{2} \frac{1}{R_{\tilde{W}}} \\
R_{\tilde{W}}=\int_{0}^{1} d y\left[1+\frac{1}{3}\left(\frac{M_{1}}{M_{2}}\right)^{3 / 2} e^{\frac{x_{f}}{y}\left(\frac{M_{2}}{M_{1}}-1\right)}\right]^{-2} \simeq\left(\frac{3}{4}\right)^{2} e^{-\xi_{\tilde{W}} x_{f}\left(\frac{M_{2}}{M_{1}}-1\right)},
\end{gathered}
$$

with $\xi_{\tilde{W}} \simeq 1$.7. This result well describes the dependence of $\Omega_{\tilde{B} \tilde{W}}$ on the $\tilde{B} / \tilde{W}$ mass ratio. Notice that the mixing angle $\theta$ is typically rather small in most of the parameter space under consideration, and that the dependence of the relic abundance on $\theta$ is modest (for small $\theta$ ), see fig. 3. Therefore eq. (55) is a good approximation in most of the well-tempered $\tilde{B} / \tilde{W}$ region.

The expressions of the annihilating cross section in the case of well-tempered $\tilde{B} / \tilde{H}$ are rather long and we do not show them here. However, the result becomes particularly simple in the case of very small mixing angles (neglecting the small $p$-wave annihilation into Higgs fields), where the relic abundance is determined by Higgsino annihilation. In this case, we find

$$
\begin{gathered}
\Omega_{\tilde{H}} h^{2}=0.10\left(\frac{\mu}{1 \mathrm{TeV}}\right)^{2} \frac{1}{R_{\tilde{H}}} \\
R_{\tilde{H}}=\int_{0}^{1} d y\left[1+\frac{1}{4}\left(\frac{M_{1}}{\mu}\right)^{3 / 2} e^{\frac{x_{f}}{y}\left(\frac{\mu}{M_{1}}-1\right)}\right]^{-2} \simeq\left(\frac{4}{5}\right)^{2} e^{-\xi_{\tilde{H}} x_{f}\left(\frac{\mu}{M_{1}}-1\right)},
\end{gathered}
$$

with $\xi_{\tilde{H}} \simeq 1.5$. The effect of the mixing angle is more important for well-tempered $\tilde{B} / \tilde{H}$ rather than $\tilde{B} / \tilde{W}$, since $\theta_{ \pm}$is parametrically larger than $\theta$ by a factor $\mu / M_{Z}$, see eqs. (10) and (19). For this reason, eq. (157) has a more limited applicability. However, in some cases, the $\tilde{B} / \tilde{H}$ mixing effects becomes negligible; this happens, for instance, when $\mu$ and $M_{1}$ have opposite signs and $\tan \beta$ is small.

In the limit in which the mass difference among neutralino states is sufficiently large and coannihilation can be neglected, the annihilation cross section for the well-tempered $\tilde{B} / \tilde{H}$ is described by

$$
\begin{array}{r}
\sigma_{\tilde{B} \tilde{B}}=\sigma_{\tilde{B} \tilde{B}}^{(s)}+\sigma_{\tilde{B} \tilde{B}}^{(p)} \\
\sigma_{\tilde{B} \tilde{B}}^{(s)}=\frac{g^{4}\left(\theta_{-}^{4}+\theta_{+}^{4}\right) M_{1}^{2}}{64 \pi\left(\mu^{2}+M_{1}^{2}\right)}\left(3+2 t_{W}^{2}+t_{W}^{4}\right)
\end{array}
$$




$$
+\frac{g^{4} \theta_{-}^{2} \theta_{+}^{2}}{128 \pi}\left[\frac{77 \mu^{4}-26 \mu^{2} M_{1}^{2}-39 M_{1}^{4}}{\left(\mu^{2}+M_{1}^{2}\right)^{3}}+\frac{2}{M_{1}^{2}}\left(7 t_{W}^{4}-6 t_{W}^{2}+3\right)\right]
$$

where $\sigma_{\tilde{B} \tilde{B}}^{(p)}$ is given in eq. (152) and $\theta_{ \pm}$are defined in eq. (10).

\section{Appendix B}

Here we give the analytic expressions of the well-tempered neutralino masses and mixing angles in the case of complex soft terms. In general the parameter $M_{1,2}$ and $\mu$ are expected to be complex, although their phases are constrained by measurements of electric dipole moments 38]. Maximal phases are allowed when squarks and sleptons are very heavy [17, 36], as in the case of Split Supersymmetry.

Consider the Higgs/Higgsino/gaugino sector. The theory has 4 (potentially) complex parameters: $M_{1}, M_{2}, \mu$, and $B_{\mu}$. By redifining the phases of $\tilde{B}, \tilde{W}, \tilde{H}_{u, d}$, and the two Higgs fields, under the constraint of keeping real the gauge coupling constants in the $H$ $\tilde{H}-\tilde{B}$ and $H-\tilde{H}-\tilde{W}$ vertices, the complex parameters transform as $B_{\mu} \rightarrow e^{i \gamma} B_{\mu}, \mu \rightarrow e^{i \tilde{\gamma}} \mu$, $M_{1,2} \rightarrow e^{i(\gamma-\tilde{\gamma})} M_{1,2}$. Therefore the invariants under phase reparametrization $\operatorname{are} \arg \left(M_{1}^{*} M_{2}\right)$ and $\arg \left(B_{\mu}^{*} \mu M_{1,2)}\right.$, but only 2 out of 3 are independent. Since after the minimization of the Higgs potential the phase of $B_{\mu}$ corresponds to the phase of $s_{2 \beta}$, we choose an independent phases

$$
\varphi_{1} \equiv \arg \left(s_{2 \beta} \mu M_{1}\right), \quad \varphi_{2} \equiv \arg \left(s_{2 \beta} \mu M_{2}\right)
$$

In the following, we choose to work in the standard basis where both Higgs vacuum expectation values are real. Notice that, in the limit of large $\tan \beta$, the invariants in eq. (61) (and the dependence on the sign of $\mu$ ) vanish, and only the relative gaugino-mass phase $\arg \left(M_{1}^{*} M_{2}\right)$ is physical.

\section{Well-Tempered Bino/Wino}

At the leading order in the $1 / \mu$ expansion, the unitary matrix $N$ that diagonalizes the neutralino mass matrix in the $\tilde{B} / \tilde{W}$ sector, according to $N \mathcal{M} N^{T}$, is given by

$$
N=D\left(\begin{array}{cc}
1 & -\theta \\
\theta^{*} & 1
\end{array}\right), \quad \theta=\frac{s_{2 W} s_{2 \beta} M_{Z}^{2}\left(M_{1} \mu+M_{2}^{*} \mu^{*}\right)}{2|\mu|^{2}\left(\left|M_{2}\right|^{2}-\left|M_{1}\right|^{2}\right)} .
$$

The diagonal matrix $D_{i j}=\delta_{i j} \sqrt{\left|m_{\chi_{i}}\right| / m_{\chi_{i}}}$ removes the phases of the mass eigenvalues, to make them real and positive. The absolute values of the mass eigenvalues for neutral and charged states are

$$
\left|m_{\chi_{1}}\right|=\left|M_{1}\right|-\frac{s_{W}^{2} s_{2 \beta} c_{\varphi_{1}} M_{Z}^{2}}{|\mu|}, \quad\left|m_{\chi_{2}}\right|=\left|m_{\chi^{+}}\right|=\left|M_{2}\right|-\frac{c_{W}^{2} s_{2 \beta} c_{\varphi_{2}} M_{Z}^{2}}{|\mu|} .
$$


Notice how the dependence on gaugino-mass phases always appears in the form of the invariants under phase reparametrization defined in eq. (61). Equations (62)- (63) interpolate between the two cases of equal and opposite signs for the gaugino masses, considered in the text. In particular, near the well-tempered condition, eq. (62) gives

$$
|\theta|=\frac{s_{2 W} s_{2 \beta} M_{Z}^{2}}{2|\mu|\left(\left|M_{2}\right|-\left|M_{1}\right|\right)} \sqrt{\frac{1+c_{\varphi_{1}+\varphi_{2}}}{2}} .
$$

This shows that $|\theta|$ vanishes only in the particular case in which $M_{1}$ and $M_{2}$ are real and have opposite sign.

\section{Well-Tempered Bino/Higgsino}

At the leading order, the unitary matrix $N$ that diagonalizes the neutralino mass matrix according to $N \mathcal{M} N^{T}$, in the $\tilde{B} / \tilde{H}$ sector is

$$
\begin{gathered}
N=D\left(\begin{array}{ccc}
1 & \theta_{+} & \theta_{-} \\
-e^{-i \phi_{+}} \theta_{+}^{*} & \cos (\alpha) & i \sin (\alpha) \\
-e^{-i \phi_{-}} \theta_{-}^{*} & i \sin (\alpha) & \cos (\alpha)
\end{array}\right) \\
\theta_{ \pm}=\frac{s_{W} M_{Z}\left(s_{\beta} \pm c_{\beta}\right)\left(\mu^{*} \pm M_{1}\right)}{\sqrt{2}\left(|\mu|^{2}-\left|M_{1}\right|^{2}\right)} \\
\tan \alpha=\frac{2 \operatorname{Im}\left(\theta_{+}^{*} \theta_{-}\right)}{\left|\theta_{+}\right|^{2}-\left|\theta_{-}\right|^{2}}, \quad \sin \phi_{ \pm}=-\sin \alpha \operatorname{Re} \frac{\theta_{\mp}}{\theta_{ \pm}} .
\end{gathered}
$$

The diagonal matrix $D$ is defined as above, and the absolute values of the mass eigenvalues are

$$
\begin{gathered}
\left|M_{1}\right|-s_{W}^{2} M_{Z}^{2}\left(\frac{\left|M_{1}\right|+|\mu| s_{2 \beta} c_{\varphi_{1}}}{|\mu|^{2}-\left|M_{1}\right|^{2}}\right) \\
|\mu|+\frac{s_{W}^{2} M_{Z}^{2}}{2\left(|\mu|^{2}-\left|M_{1}\right|^{2}\right)}\left[|\mu|+\left|M_{1}\right| s_{2 \beta} c_{\varphi_{1}} \pm \sqrt{\left(|\mu| s_{2 \beta}+\left|M_{1}\right| c_{\varphi_{1}}\right)^{2}+\left|M_{1}\right|^{2} c_{2 \beta}^{2} s_{\varphi_{1}}^{2}}\right]
\end{gathered}
$$

Notice an important difference with the case of real soft parameters. The states $\tilde{H}_{1,2}$ defined in sect. 2 are not approximate mass eigenstates, for a generic phase $\varphi_{1}$, but they are rotated by an angle $\alpha$, which is typically of order unity. In the particular cases $\varphi_{1}=0, \pi$ (corresponding to real $M_{1}$ and $\mu$, with equal or opposite signs), $\theta_{ \pm}$are real, and $\alpha=\phi_{ \pm}=0$, see eqs. (66) - (67). In this case the mixing between $\tilde{H}_{1}$ and $\tilde{H}_{2}$ appears only at $\mathcal{O}\left(M_{Z}^{2}\right)$, as shown in eq. (11). The mass eigenvalues given in eqs. (68)- (69) reduce to

$$
\left|M_{1}\right|-s_{W}^{2} M_{Z}^{2}\left(\frac{\left|M_{1}\right|+|\mu| s_{2 \beta} c_{\varphi_{1}}}{|\mu|^{2}-\left|M_{1}\right|^{2}}\right), \quad|\mu|+\frac{s_{W}^{2} M_{Z}^{2}\left(1 \pm s_{2 \beta}\right)}{2\left(|\mu| \mp c_{\varphi_{1}}\left|M_{1}\right|\right)},
$$

where $c_{\varphi_{1}}= \pm 1$, when $M_{1}$ and $\mu$ have equal or opposite signs, respectively. This result corresponds to eq. (9). 


\section{References}

[1] G. Hinshaw et al., Astrophys. J. Suppl. 148 (2003) 135 arXiv:astro-ph/0302217; C. J. MacTavish et al., arXiv:astro-ph/0507503 A. G. Sanchez et al., arXiv:astro-ph/0507583

[2] S. Eidelman et al. [Particle Data Group], Phys. Lett. B 592 (2004) 1.

[3] H. Goldberg, Phys. Rev. Lett. 50 (1983) 1419; J. R. Ellis, J. S. Hagelin, D. V. Nanopoulos, K. A. Olive and M. Srednicki, Nucl. Phys. B 238 (1984) 453.

[4] LEP2 SUSY Working Group, LEPSUSYWG/04-01.1 (2004).

[5] J. R. Ellis, T. Falk and K. A. Olive, Phys. Lett. B 444 (1998) 367 arXiv:hep-ph/9810360; J. R. Ellis, T. Falk, K. A. Olive and M. Srednicki, Astropart. Phys. 13 (2000) 181 [Erratum-ibid. 15 (2001) 413] arXiv:hep-ph/9905481.

[6] K. Griest and D. Seckel, Phys. Rev. D 43 (1991) 3191.

[7] M. Drees and M. M. Nojiri, Phys. Rev. D 47 (1993) 376 arXiv:hep-ph/9207234;

L. Roszkowski, R. Ruiz de Austri and T. Nihei, JHEP 0108 (2001) 024 arXiv:hep-ph/0106334; A. Djouadi, M. Drees and J. L. Kneur, Phys. Lett. B 624 (2005) 60 arXiv:hep-ph/0504090.

[8] G. F. Giudice, E. W. Kolb and A. Riotto, Phys. Rev. D 64 (2001) 023508 arXiv:hep-ph/0005123.

[9] J. L. Feng, A. Rajaraman and F. Takayama, Phys. Rev. Lett. 91 (2003) 011302 arXiv:hep-ph/0302215; J. L. Feng, A. Rajaraman and F. Takayama, Phys. Rev. D 68, 063504 (2003) arXiv:hep-ph/0306024; J. R. Ellis, K. A. Olive, Y. Santoso and V. C. Spanos, Phys. Lett. B 588 (2004) 7 arXiv:hep-ph/0312262; J. L. Feng, S. f. Su and F. Takayama, Phys. Rev. D 70 (2004) 063514 arXiv:hep-ph/0404198; J. L. Feng, S. Su and F. Takayama, Phys. Rev. D 70 (2004) 075019 arXiv:hep-ph/0404231; L. Roszkowski, R. Ruiz de Austri and K. Y. Choi, JHEP 0508, 080 (2005) arXiv:hep-ph/0408227.

[10] D. G. Cerdeno, K. Y. Choi, K. Jedamzik, L. Roszkowski and R. Ruiz de Austri, arXiv:hep-ph/0509275.

[11] L. Covi, H. B. Kim, J. E. Kim and L. Roszkowski, JHEP 0105 (2001) 033 arXiv:hep-ph/0101009; K. Kohri, M. Yamaguchi and J. Yokoyama, Phys. Rev. D 72 (2005) 083510 arXiv:hep-ph/0502211. 
[12] K. L. Chan, U. Chattopadhyay and P. Nath, Phys. Rev. D 58 (1998) 096004 arXiv:hep-ph/9710473.

[13] J. L. Feng, K. T. Matchev and T. Moroi, Phys. Rev. D 61 (2000) 075005 arXiv:hep-ph/9909334.

[14] S. Mizuta and M. Yamaguchi, Phys. Lett. B 298 (1993) 120 arXiv:hep-ph/9208251; U. Chattopadhyay, D. Choudhury, M. Drees, P. Konar and D. P. Roy, arXiv:hep-ph/0508098.

[15] N. Arkani-Hamed and S. Dimopoulos, JHEP 0506 (2005) 073 arXiv:hep-th/0405159.

[16] G. F. Giudice and A. Romanino, Nucl. Phys. B 699 (2004) 65 [Erratum-ibid. B 706 (2005) 65] arXiv:hep-ph/0406088.

[17] N. Arkani-Hamed, S. Dimopoulos, G. F. Giudice and A. Romanino, Nucl. Phys. B 709 (2005) 3 arXiv:hep-ph/0409232.

[18] L. Randall and R. Sundrum, Nucl. Phys. B 557 (1999) 79 arXiv:hep-th/9810155.

[19] G. F. Giudice, M. A. Luty, H. Murayama and R. Rattazzi, JHEP 9812 (1998) 027 arXiv:hep-ph/9810442.

[20] T. Moroi and L. Randall, Nucl. Phys. B 570 (2000) 455 arXiv:hep-ph/9906527.

[21] T. Gherghetta, G. F. Giudice and J. D. Wells, Nucl. Phys. B 559 (1999) 27 arXiv:hep-ph/9904378.

[22] J. L. Feng, K. T. Matchev and F. Wilczek, Phys. Lett. B 482 (2000) 388 arXiv:hep-ph/0004043.

[23] H. Baer, A. Mustafayev, S. Profumo, A. Belyaev and X. Tata, JHEP 0507 (2005) 065 arXiv:hep-ph/0504001.

[24] A. Pierce, Phys. Rev. D 70 (2004) 075006 arXiv:hep-ph/0406144.

[25] A. Masiero, S. Profumo and P. Ullio, Nucl. Phys. B 712 (2005) 86 arXiv:hep-ph/0412058.

[26] H. Baer, A. Mustafayev, E. K. Park and S. Profumo, JHEP 0507 (2005) 046 arXiv:hep-ph/0505227; H. Baer, T. Krupovnickas, A. Mustafayev, E. K. Park, S. Profumo and X. Tata, arXiv:hep-ph/0511034. 
[27] A. Birkedal-Hansen and B. D. Nelson, Phys. Rev. D 64, 015008 (2001) arXiv:hep-ph/0102075; ; A. Birkedal-Hansen and B. D. Nelson, Phys. Rev. D 67, 095006 (2003) arXiv:hep-ph/0211071.

[28] P. Gondolo, J. Edsjo, P. Ullio, L. Bergstrom, M. Schelke and E. A. Baltz, JCAP 0407 (2004) 008 arXiv:astro-ph/0406204.

[29] R. Barbieri, M. Frigeni and G. F. Giudice, Nucl. Phys. B 313 (1989) 725.

[30] D. M. Pierce, J. A. Bagger, K. T. Matchev and R. j. Zhang, Nucl. Phys. B 491 (1997) 3 arXiv:hep-ph/9606211.

[31] G. F. Giudice and A. Masiero, Phys. Lett. B 206 (1988) 480.

[32] J. D. Wells, Phys. Rev. D 71 (2005) 015013 arXiv:hep-ph/0411041.

[33] J. Kaplan, to appear.

[34] M. Toharia and J. D. Wells, arXiv:hep-ph/0503175; P. Gambino, G. F. Giudice and P. Slavich, Nucl. Phys. B 726 (2005) 35 arXiv:hep-ph/0506214.

[35] T. Goldman and H. Haber, Physica 15D (1985) 181; E. Chikovani, V. Kartvelishvili, R. Shanidze and G. Shaw, Phys. Rev. D 53 (1996) 6653 arXiv:hep-ph/9602249; E. Bouhova-Thacker, V. Kartvelishvili and A. Small, Nucl. Phys. Proc. Suppl. 133 (2004) 122; K. Cheung and W. Y. Keung, Phys. Rev. D 71 (2005) 015015 arXiv:hep-ph/0408335.

[36] N. G. Deshpande and J. Jiang, Phys. Lett. B 615 (2005) 111 arXiv:hep-ph/0503116; D. Chang, W. F. Chang and W. Y. Keung, Phys. Rev. D 71 (2005) 076006 arXiv:hep-ph/0503055; G. F. Giudice and A. Romanino, arXiv:hep-ph/0510197.

[37] M. Tegmark, A. Aguirre, M. Rees and F. Wilczek, arXiv:astro-ph/0511774.

[38] M. Pospelov and A. Ritz, Annals Phys. 318 (2005) 119 arXiv:hep-ph/0504231. 